\title{
Sen1 is a master regulator of transcription-driven conflicts
}

Umberto Aiello ${ }^{1}$, Drice Challal ${ }^{1,2}$, Griselda Wentzinger $^{1}$, Armelle Lengronne $^{3}$, Rowin Appanah $^{4}$, Philippe Pasero ${ }^{3}$, Benoit Palancade ${ }^{1}$, Domenico Libri ${ }^{1 *}$

* to whom correspondence should be addressed: domenico.libri@ijm.fr

${ }^{1}$ Université de Paris, CNRS, Institut Jacques Monod, F-75013 Paris, France

${ }^{2}$ present address : Institut de Biologie Intégrative de la Cellule UMR9198 CEA, CNRS, Université Paris-Saclay, F-91198 Gif-sur-Yvette, France

${ }^{3}$ Institut de Génétique Humaine, CNRS, Université de Montpellier, Montpellier, France

${ }^{4}$ Genome Damage and Stability Centre, School of Life Sciences, University of Sussex, Falmer, Brighton BN1 9RQ, UK 


\begin{abstract}
Cellular homeostasis requires the coordination of several machineries concurrently engaged on the DNA. Wide-spread transcription can interfere with other processes and transcription-replication conflicts (TRCs) threaten genome stability. The conserved Sen1 helicase terminates non-coding transcription, but also interacts with the replisome and reportedly resolves genotoxic R-loops. Sen1 prevents genomic instability but how this relates to its molecular functions remains unclear. We generated high-resolution, genome-wide maps of transcription-dependent conflicts and R-loops using a Sen1 mutant that has lost interaction with the replisome but is termination proficient. We show that Sen1 removes RNA polymerase II at TRCs within genes and the rDNA, but also at sites of transcription-transcription conflicts under physiological conditions, thus qualifying as a "master regulator of conflicts". We demonstrate that genomic stability is only affected by Sen 1 mutation when, in addition to its role at the replisome, termination of non-coding transcription or R-loop removal are additionally compromised.
\end{abstract}

Keywords: transcription; replication; transcription-replication conflicts (TRCs); Sen1; RNase H; R-loops; genome stability; non-coding transcription; H-CRAC 


\section{INTRODUCTION}

The DNA is the shared workspace synchronously used by many cellular machineries that are essential for the correct expression, maintenance, repair, and transmission of the genetic information. Because they work concurrently, the orchestration of these activities must be accurately coordinated, both in time and space, to avoid interferences that might ultimately lead to mis-expression or corruption of the genetic content. Seemingly at odd with these necessities, transcription occupies the virtual integrity of the genome. RNA Polymerase II (RNAPII) transcribes largely beyond the limits dictated by apparent physiological significance, a phenomenon dubbed pervasive transcription. Robust and accurate mechanisms are required for limiting conflicts or solving them, but the actors involved, and their mode of action are not fully understood.

Transcription-replication conflicts (TRCs) are of marked interest in the crowded genomic landscape as they can generate genomic instability and jeopardize the faithful transmission of genetic information. The inherent stability of transcription elongation complexes is sufficient for inducing stalling of replication forks, a condition that has potential to generate DNA damage, in particular when associated to the formation of R-loops. These structures are characterized by a peculiar topological arrangement in which the nascent RNA associates to its DNA template, leaving unpaired the cognate DNA strand. R-loops have important physiological functions in the generation of antibody diversity, and other processes (for a review see: Feng et al., 2020), but their non-physiological accumulation is generally considered genotoxic.

The helicase Sen1 has a particular place in the orchestration of transcription and replication activities. Within the Nrd1-Nab3-Sen1 (NNS) complex, it has an essential role in controlling transcription termination at thousands of genes producing non-coding RNAs, some of which are stable and functional, such as snoRNAs involved in rRNA maturation, while others are unstable (Cryptic Unstable Transcripts, CUTs) and degraded by the nuclear exosome rapidly after transcription (Steinmetz et al., 2006; Hazelbaker et al., 2012; Porrua and Libri, 2013; Schaughency et al., 2014). Failures in NNS-dependent termination by depletion of Nrd1 
has been shown to generate extended transcription events that affect the expression of neighboring genes, thus altering the overall transcriptional homeostasis of the cell (Schulz et al., 2013). Besides a role in limiting the chances of conflicts by restricting pervasive transcription, Sen1 has been proposed to work directly at sites of TRCs. Sen1 loss-of-function mutants, or strains in which the protein has been depleted, display genomic instability phenotypes revealed by increased mitotic recombination between direct repeats, synthetic lethality with DNA repair mutants and Rad52 foci accumulation (Mischo et al., 2011). These effects have been attributed to the defective resolution of R-loops at TRCs in the light of increased fork stalling at sites of convergent transcription and replication and the genomic colocalization of Sen1 and replication forks (Alzu et al., 2012). Indeed, increased R-loop levels have been detected in these Sen1 loss-of-function genetic backgrounds especially during Sphase (Mischo et al., 2011; San Martin-Alonso et al., 2021), which led to the proposal that Sen1, by virtue of its helicase activity, resolves R-loops that are formed at TRCs. However, in these mutant contexts, transcription termination of many ncRNA genes is affected, with potential effects on the phenotypes observed. Thus, in the absence of Sen1 it is conceivable that rather than (or in addition to) the defective resolution of constitutively formed R-loops, more R-loops are formed as a consequence of the generally higher transcriptional readthrough, which entails increased chance of conflicts. Although genomic instability was not observed in other mutants of the NNS complex that have termination defects (Costantino and Koshland, 2018; Mischo et al., 2011), it remains possible that the phenotypes associated to Sen1 loss-of-function originate from the synthetic association of increased transcriptional challenges and failure to resolve conflicts. Disentangling the contributions of these potential synthetic effects is paramount for understanding the function of Sen1 in maintaining genomic stability.

We have recently reported the physical interaction of Sen1 with the Ctf4 and Mrc1 replisome components and characterized a mutant, sen1-3, that loses this interaction (Appanah et al., 2020). Sen1-3 cells have a minor growth phenotype and, importantly, no transcription termination defects at NNS target genes. However, this mutation induces lethality 
in the absence of the two yeast RNases H, Rnh1 and Rnh201/202/203, which, together with other genetic interactions with mutants of the fork stalling signaling pathway (Appanah et al., 2020), underscores the physiological relevance of the interaction of Sen1 with the replisome. Importantly, RNase $\mathrm{H} 1$ and $\mathrm{H} 2$ are redundantly involved in the degradation of the RNA moiety of R-loops, which might mechanistically underlie a functional connection with Sen1 at TRCs.

In the course of a separate study we have shown that Sen1 is required for a back-up mechanism of RNAPIII release when primary termination has failed (Xie et al., 2021). The interactions of Sen1 with the replisome and RNAPIII are mutually exclusive, which implicates the existence of two distinct Sen1-containing complexes presumably with different functional roles. Interestingly, however, both interactions are impaired by the sen1-3 mutation, which likely alters a shared region of interaction (Xie et al., 2021). These findings indicate that the sen1-3 mutant allows untangling the function of Sen1 in NNS-dependent termination from its functions at the replisome and RNAPIII transcription.

Here we first addressed the functional impact of the sen1-3 mutation on transcriptionreplication conflicts under conditions in which neither transcription nor replication are altered. Prompted by the strong genetic interaction with RNases $\mathrm{H}$ we also studied the role of these enzymes at sites of conflicts and the impact of R-loops. To this aim we generated high resolution transcription maps in different phases of the cell cycle in sen1-3 cells and in the absence of RNases $\mathrm{H}$. We also devised a novel methodology to detect $\mathrm{R}$-loops in vivo with high sensitivity and unprecedented resolution. We show that Sen1 is required for the efficient removal of RNAPII at many TRC sites within genes, but also at the rDNA, where it collaborates with RNases $\mathrm{H}$. Surprisingly, when non-coding transcription is correctly terminated, loss of the interaction of Sen 1 with the replisome does not cause increased R-loop accumulation, mitotic recombination or DNA damage as observed in sen 1 loss-of-function mutants. We demonstrate that increased DNA damage observed in these mutants requires both the lack of Sen1 interaction with the replisome and the transcription termination defects at non-coding RNA genes. Interestingly, we show that Sen1 also functions at many other genomic sites to remove RNAPII at sites of conflicts with RNAPIII and possibly RNAPI. We propose a model according 
bioRxiv preprint doi: https://doi.org/10.1101/2022.02.09.479708; this version posted February 10, 2022. The copyright holder for this preprint (which was not certified by peer review) is the author/funder, who has granted bioRxiv a license to display the preprint in perpetuity. It is made available under aCC-BY-NC-ND 4.0 International license.

to which Sen1 functions as a "master regulator of conflicts" in the genome, and we demonstrate that this function is independent from its role in terminating non-coding transcription. 


\section{RESULTS}

\section{Sen1 depletion leads to major genome-wide alterations in the transcriptome}

To assess directly the possible contribution of transcription termination defects to the genomic instability phenotypes observed in Sen1 loss-of-function mutants, we first directly gauged the extent of alterations in coding and non-coding RNAPII transcriptional activity under defective Sen1 function, which was previously investigated only to a limited extent (Schaughency et al., 2014). In this former report Sen1 was depleted by the anchor away methodology (Haruki et al., 2008), which, in our hands, did not induce major growth defects, possibly because of partial Sen1 depletion. For consistency with the data in this and other studies (Costantino and Koshland, 2018; San Martin-Alonso et al., 2021), we generated high resolution transcription maps using RNAPII CRAC (Crosslinking Analysis of cDNAs, Bohnsack et al., 2012; Candelli et al., 2018) upon depletion of Sen1 by the auxin degron system (Nishimura et al., 2009). In the CRAC methodology, the nascent RNA is crosslinked to Rpb1, the largest subunit of RNAPII, which is purified under denaturing conditions, thus limiting copurification of associated, non-nascent transcripts. Sequencing of the crosslinked RNA provides the position of the polymerase with directionality and high resolution.

Depletion of Sen 1 by addition of auxin induced the expected transcription termination defects at canonical NNS targets (CUT and snoRNA genes, Figures S1A, left panel, and S1B), but not at mRNA coding genes (Figure S1A, right panel), consistent with the Schaugency et al. study (Schaughency et al., 2014).

Defective RNAPII release at NNS targets could ultimately result in a global redistribution of polymerases with increased persistency in some regions, as well as depletions in others, possibly because of transcription interference. To estimate the occurrence of global transcriptional changes in an unbiased manner, we first computed the RNAPII CRAC signal in intergenic regions divided in $200 \mathrm{nt}$, non-overlapping windows with the exclusion of mRNAcoding genes, the rDNA and tRNA genes to first focus on regions of direct Sen1 action. Scatter plots of these values revealed a dramatic alteration of intergenic transcription upon Sen1 depletion compared to wild-type cells (Figures 1A, compare left and right). Similar 
bioRxiv preprint doi: https://doi.org/10.1101/2022.02.09.479708; this version posted February 10,2022 . The copyright holder for this preprint (which was not certified by peer review) is the author/funder, who has granted bioRxiv a license to display the preprint in perpetuity. It is made available under aCC-BY-NC-ND 4.0 International license.

transcription alterations were observed upon auxin-dependent depletion of another NNS component, Nrd1, indicating that they are linked to a transcription termination defect (Figure $\mathrm{S1C})$.

\section{Figure 1}

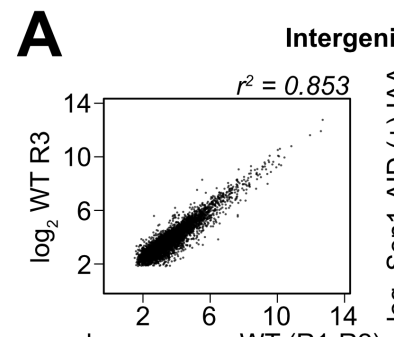

$\log _{2}$ average WT (R1-R2)

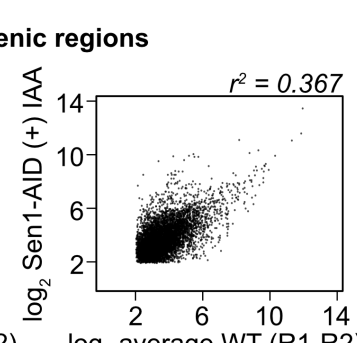

$\log _{2}$ average WT (R1-R2)
B Coding genes

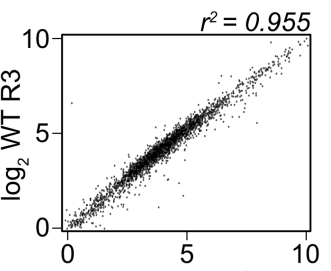

$\log _{2}$ average WT (R1-R2)

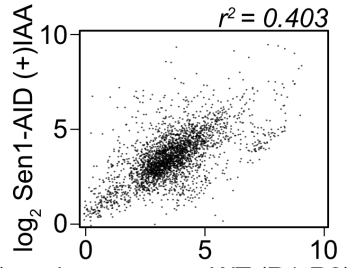

$\log _{2}$ average WT (R1-R2)

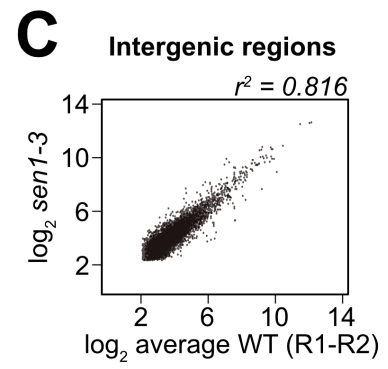

Doding genes

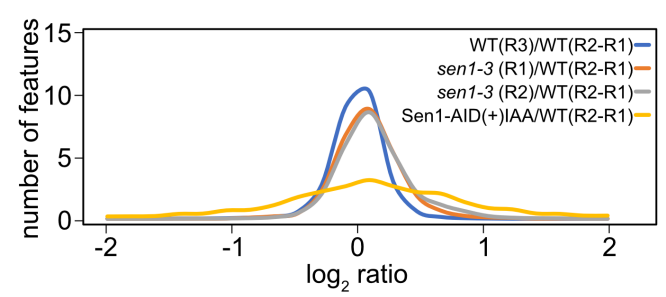

Figure 1: Major alterations in the transcriptome upon Sen1 depletion

A) Scatter plots of RNAPII CRAC $\log _{2}$ values computed in non-overlapping $200 \mathrm{nt}$ bins relative to the average of two wild-type replicates (WT, R1-R2) used as a common reference in all panels. Only the W strand was used to exclude background signals derived from contaminating rRNA. mRNA-coding and tRNA genes have also been excluded from this analysis. The scatter plot of RNAPII CRAC signals derived from Sen1 depleted cells is presented (right), together with the scatter plot of another wild-type replicate (left, WT R3) for comparison and visual assessment of reproducibility. Bins containing a low number of reads $\left(\log _{2}<2\right)$ have been excluded from the analysis for clarity. B) As in A but RNAPII CRAC average signal $\left(\log _{2}\right)$ from mRNA coding genes have been computed. C) As in A, right panel, intergenic RNAPII CRAC values from sen1-3 cells are compared to the wildtype reference. D) Distribution of $\log _{2}$ ratios of average RNAPII CRAC signals detected on mRNA coding genes. Data from two independent sen1-3 replicates, evaluated relative to the wild-type reference. An additional wild-type replicate is shown for comparison together with the distribution obtained upon Sen1 depletion. 
Deregulation of non-coding transcription might induce significant effects on the proteincoding transcriptome, which are susceptible to influence normal cellular physiology. Therefore, we also analyzed the transcription levels of mRNA-coding genes in Sen1-AID strains by RNAPII CRAC. The gene expression program was also significantly modified upon Sen1 depletion (Figure 1B). These effects were generally recapitulated upon depletion of Nrd1, the two datasets showing a high level of correlation $\left(r^{2}=0.726\right.$, Figure S1D). Because we did not observe termination defects at genes with altered expression (and at mRNA genes in general, Figure S1B, first panel), it is likely that these effects are at least partially due to a cellular response to the perturbations introduced by Sen1 (and Nrd1) depletion. Notably, many stress genes are activated and ribosomal protein genes downregulated, possibly suggesting the occurrence of a marked stress response (see Discussion).

These results demonstrate that loss of full Sen1 function has a major impact on the distribution of transcription events genome-wide and on gene expression. These changes are expected to alter the wild-type landscape of TRCs and might contribute significantly to the genomic instability phenotypes observed in Sen1 loss-of-function mutants.

\section{Interaction of Sen1 with the replisome is required to solve TRCs in the 5'-end of} genes.

In the light of the above considerations, we sought to analyze the role of Sen 1 in genomic stability in a context that would be more physiological in terms of transcriptional landscape. Genetic data strongly support the notion that the role of Sen1 at TRCs is mechanistically linked to its interaction with the replisome, and therefore we turned to the use of the sen1-3 mutant that loses this interaction, but is proficient for NNS termination (Appanah et al., 2020). In sharp contrast to what observed upon depletion of Sen1, affecting the interaction of Sen1 with the replisome does not alters significantly the coding and non-coding transcriptome (Figures 1C and 1D), validating the use of the sen1-3 mutant for specifically focusing on the role of Sen1 at TRCs. 
bioRxiv preprint doi: https://doi.org/10.1101/2022.02.09.479708; this version posted February 10, 2022. The copyright holder for this

preprint (which was not certified by peer review) is the author/funder, who has granted bioRxiv a license to display the preprint in perpetuity. It is made available under aCC-BY-NC-ND 4.0 International license.

Figure 2
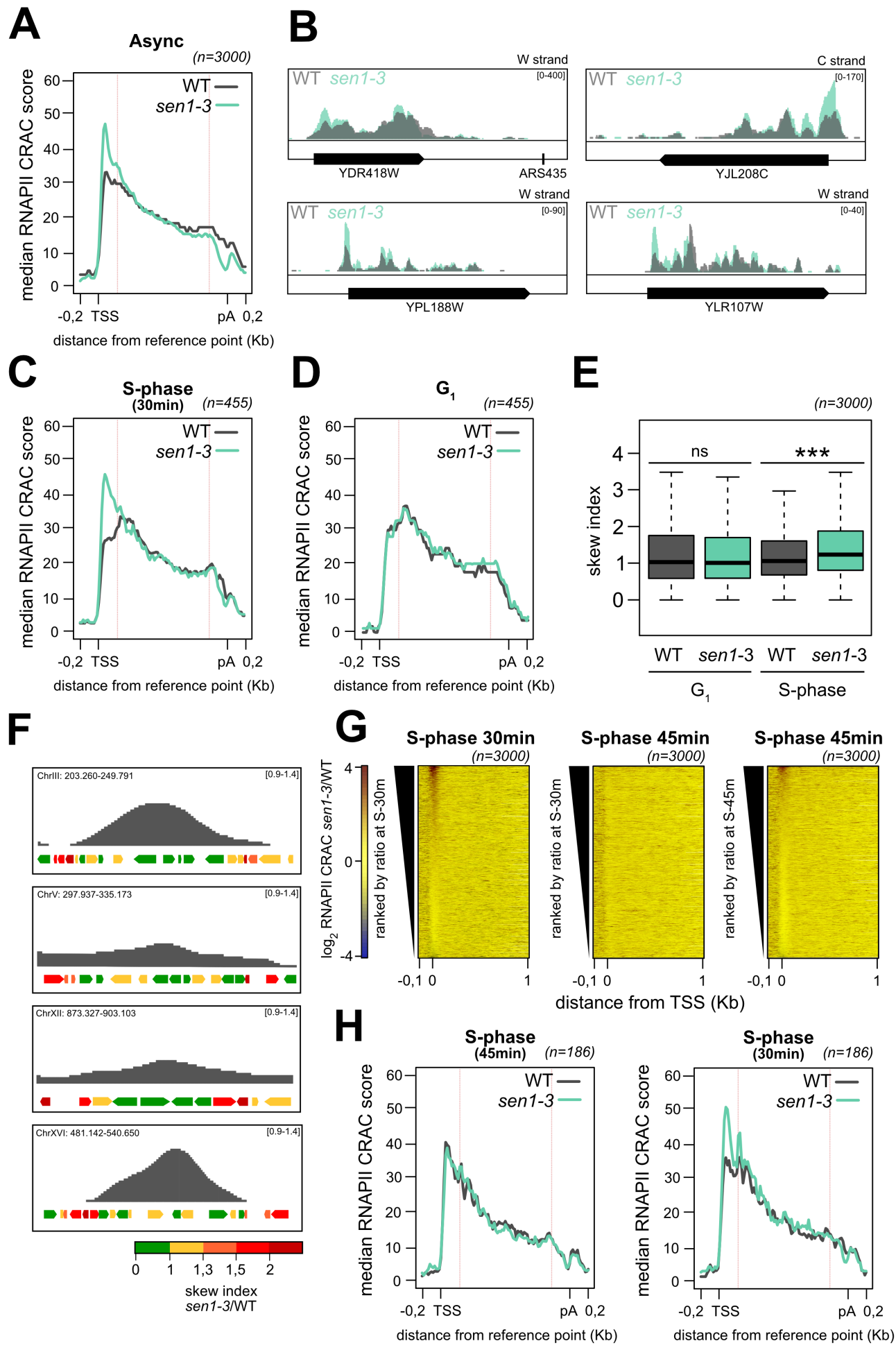
Figure 2: Sen1 promotes release of RNAPII from the 5'-end of genes undergoing replication.

A) Metagene analysis of the RNAPII distribution at coding genes aligned at their Transcription Start Site (TSS) and at their poly-Adenylation (pA) site in wild-type (WT) and sen1-3 cells grown asynchronously. The 3000 genes with the highest expression (as determined by RNAPII CRAC) have been included in the analysis. Values on the y-axis correspond to the median coverage. Genes are only scaled in the interval delimited by red lines. B) Integrative Genomics Viewer (IGV) screenshots of representative examples of coding genes illustrating RNAPII accumulation in sen1-3 cells. The overlap of RNAPII read coverage in wild-type (WT, grey) and sen1-3 cells (aqua green) is shown. The scale and the strand (W for Watson, $\mathrm{C}$ for Crick) are indicated in brackets. C) Metagene analysis as in A, but for the genes with the highest sen1-3/WT skew index ratio (i.e. higher than the mean plus one standard deviation). Analysis performed in cells synchronously released in S-phase and collected 30 min after replication onset. D) Metagene analysis performed on the same gene set as in $\mathrm{C}$ but for cells arrested in $\mathrm{G}_{1}$ by a-factor. E) Comparison of the gene skew ratio for the indicated strains in $\mathrm{G}_{1}$-arrested cells or in cells synchronously released in S-phase and collected $30 \mathrm{~min}$ after replication onset. Analysis preformed on the 3000 most expressed genes as in A. ${ }^{* * *} p<0.001$. F) Integrative Genomics Viewer (IGV) representative screenshots of replicons as detected by DNA copy number analyses for cells synchronously released in S-phase and collected after $30 \mathrm{~min}$. Genes in each region were coloured according to their skew index sen1-3/WT ratio as indicated at the bottom of the panel. Genes with the highest skew index ratio (red and orange) are located preferentially at the borders of each replicon, where replication is most likely to be ongoing. G) Heatmap analyses representing the $\log _{2}$ fold change (FC) of the RNAPII CRAC signal in the sen1-3 mutant relative to the WT for mRNA coding genes aligned at their Transcription Start Site (TSS) in S-phase at $30 \mathrm{~min}$ and $45 \mathrm{~min}$ as indicated. Genes were ranked, as indicated, according to the signals detected in the first $200 \mathrm{nt}$ after the TSS at the $30 \mathrm{~min}$ (left and central panel) or 45 min time point (right panel). H) Left: Metagene analysis as in Figure 2C, but on cells collected 45 min after replication onset and on a group of genes already replicated at the indicated time point. Right: the same group of genes are undergoing replication at $30 \mathrm{~min}$ after release in S-phase and show the characteristic 5'-end skew in sen1-3 cells.

Failure to resolve or to avoid a TRC is expected to result in slowing down or stalling of a replication fork but also to induce the accumulation of RNAPII at the site of conflict. We focused on the transcription side of the conflict and reasoned that if Sen1 is recruited at the replisome to terminate conflicting transcription, in its absence RNAPII should accumulate at these sites, thus providing a signature of Sen1-dependent conflicts.

Assuming that conflicts depend, at least to some extent, on the stochastic encounters of the two machineries, they can be expected to be more frequent in genomic regions with 
inherently higher RNAPII occupancy. In this perspective, a marked asymmetry of RNAPII distribution is clearly observed for yeast genes in wild-type cells, with higher levels in the 5'end (Figure S1A, right panel; see also Churchman and Weissman, 2011; Mayer et al., 2011) where the elongation complex is known to pause. Note that the general 5'-end proximal pausing is an inherent feature of the transcription process that is independent of replication as it is observed also in cells arrested in $\mathrm{G}_{1}$ (see below, Figure 2D).

We therefore first analysed the distribution of RNAPII on yeast genes with a particular focus on the regions of transcriptional pausing. An alteration of the RNAPII profile was clearly observed by metasite analyses and by the inspection of individual genes in asynchronously growing sen $1-3$ cells, with an increase in occupancy in the 5'-end that gradually decreases in the 3'-end (Figures 2A and 2B). In some cases, increased occupancy was also observed at other sites of RNAPII pausing (e.g., see YDR418W in Figure 2B). This pattern is not compatible with increased transcription initiation at a set of genes, which would result in a homogeneous, increase of the RNAPII CRAC signal all along these genes. Rather, it points to the occurrence of increased RNAPII pausing, mainly in the 5'-end of genes, possibly due to defective RNAPII release at sites of conflicts when Sen1 is absent from the replisome.

To assess the dependency on ongoing replication, RNAPII CRAC was performed using cells arrested in $\mathrm{G}_{1}$ and synchronously released in S-phase. The progression of replication was analysed in the same cells by DNA copy number analyses. At 30 minutes after the release in S-phase, abnormal RNAPII occupancy was observed in sen1-3 cells (Figure S2A), recapitulating what observed in asynchronous cells and, consistent with the hypothesis of a replication-dependent phenomenon, only a subset of genes was affected (see below, Figure $2 \mathrm{G})$. For a quantitative analysis of these observations, we calculated a skew index, defined as the ratio of the RNAPII CRAC score in the 5'-end [TSS; TSS+200] to the signal in a downstream region of identical length [TSS+300; TSS+500]. The skew index is expected to be poorly sensitive to changes in transcription levels, which might slightly vary in the different strains. For more robust analyses, we focused on the subset of most affected genes, identified by computing the ratio of the skew indexes from the sen $1-3$ and the wild-type cells and 
selecting features with ratios one standard deviation over the mean. This resulted in a set of 455 genes, with a marked RNAPII CRAC signal increase in sen1-3 cells slightly upstream of the canonical 5' peak detected in the wild-type (Figure 2C). Most importantly, increased RNAPII 5' occupancy was not observed in the absence of replication, when cells were arrested in $\mathrm{G}_{1}$ by the addition of alpha factor, as demonstrated by the pattern of RNAPII distribution (compare Figures 2C and 2D) and the distributions of skew indexes, which were significantly different only in S phase (Figure 2E). This conclusion also holds genome-wide, when considering the set of most expressed 3000 genes (Figure S2B). These findings indicate that replication is required for the increased occupancy of RNAPII observed in the 5'-end of genes in sen1-3 cells, supporting the notion that RNAPII is not released efficiently at TRCs when Sen1 cannot interact with the replisome.

If the replication-dependent RNAPII increase in the 5'-end of genes is linked to TRCs, the group of affected genes should be located in regions where replication is ongoing. To address this point, we divided all genes in 5 different groups based on the skew index ratio and monitored their distribution along the chromosomes in relation to the position of the replicative forks as detected by DNA copy number analyses in the same experiment. Because of population heterogeneity, the distribution of normalized DNA levels (Figures $2 \mathrm{~F}$ and S2C, see Materials and Methods) is linked to the probability that a given sequence has already been replicated (close to the center of the replicon) or is undergoing replication (close to the periphery) at the analyzed time point. The genes with the highest skew index ratios were flanking or included in virtually every active replicon but were not distributed randomly. As shown in Figures $2 \mathrm{~F}$ and $\mathrm{S} 2 \mathrm{C}$, we found that they are clearly preferentially positioned towards the borders, i.e., in regions where replication is most likely to be ongoing.

Genes in both a co-directional (CD) and head-on (HO) orientation relative to the direction of replication were equally found to be affected (Figures $2 F, S 2 C$ and S2D), as also shown by measuring the distance of the closest origins generating $\mathrm{HO}$ or $\mathrm{CD}$ replication for each affected gene (Figure S2E). This suggests that Sen1 can solve both kinds of conflicts by binding to the replisome. 
The position of TRCs is expected to change as replication progresses. Therefore, we generated additional RNAPII CRAC transcription maps at 45 min after release in S-phase and compared the results to the first transcription map that was generated at an earlier, 30 min time point. Analysis of the RNAPII CRAC signal generated at a later replication time point recapitulated the phenotype observed at the $30 \mathrm{~min}$ time point (Figure S2F and 2G, right panel), yielding a group of 439 affected genes (Figure S2G) selected following the aforementioned criteria. However, when the genes ranked for increased RNAPII occupancy at the 30 min time point were monitored for RNAPII CRAC signals at the 45 min time point (Figure 2G, compare left and middle panel), a very poor overlap, if any, was observed, consistent with the notion that TRCs are restricted to the set of genes undergoing replication at a given time point.

If RNAPII 5'-end accumulation is due to TRCs, genes that have already undergone replication at a given time point should not display increased RNAPII 5' persistency. Consistent with this notion, RNAPII CRAC analysis at a set of 186 genes selected for having been replicated at the 45 min time point according to our replication maps revealed no increase in RNAPII persistency at the 5'-end of genes in sen1-3 cells (Figure 2H, left panel).

Increased persistency of RNAPII at many genomic sites might affect replication. In agreement with the presence of a challenged replication environment, the size of early replicons as assessed by DNA copy number analysis was found to be significantly smaller in sen1-3 cells relative to the wild-type (Figure $\mathrm{S} 2 \mathrm{H}$, left panel). Interestingly, the same analysis also revealed the premature activation of late origins in the sen1-3 mutant (Figure $\mathrm{S} 2 \mathrm{H}$, right panel), which compensates the general diminished fork progression, thus explaining the similar length of S-phase observed in sen1-3 and wild-type cells (Appanah et al., 2020).

Together, these findings indicate that ongoing replication is required for the accumulation of RNAPII in the 5'-end of a set of genes, which we propose to be due to the failure to remove RNAPII from sites of TRCs when Sen1 cannot interact with the replisome. They also suggest that TRCs are frequently occurring even under physiological conditions and that the failure to efficiently prevent or resolve them alters the replication program. 
Association of Sen1 with the replisome is required for limiting RNAPII accumulation at the ribosomal replication fork barrier in S-phase.

The ribosomal DNA Replication Fork Barrier (rRFB) is a site where one replication fork stalls upstream of the DNA-bound Fob1 protein at the 3'-end of ribosomal DNA repeats (Kobayashi, 2003). This ensures that each rDNA repeat is being replicated in a co-directional fashion with RNAPI and RNAPIII transcription (Figure 3A).

\section{Figure 3}
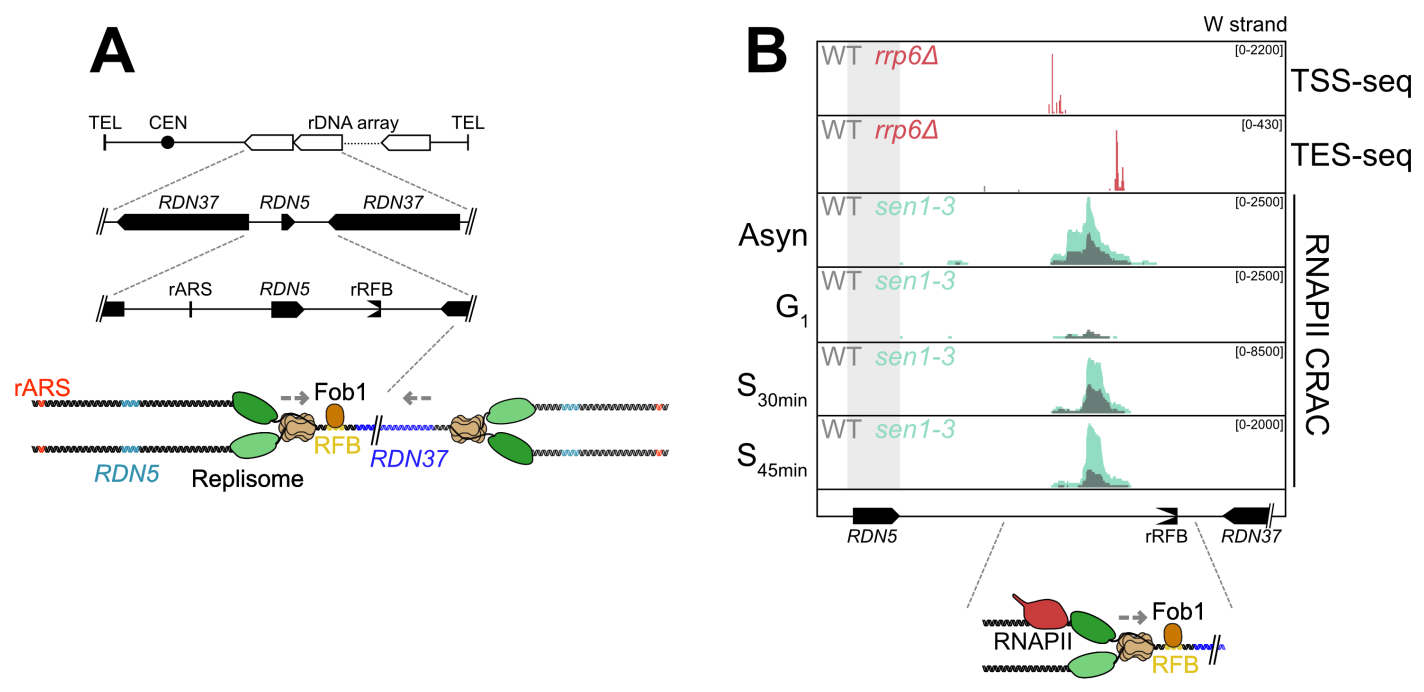

Figure 3: Association of Sen1 with the replisome is required for limiting TRCs at the replication fork barrier

A) Schematic representation of the budding yeast rDNA locus on ChrXII. The position of the replication origin (rARS) and of the rRFB are indicated relative to the RDN37 and RDN5 genes. A cartoon illustrates the process of replication in this region and the function of the Fob1 protein at the rRFB. The direction of progression for each replisome is indicated as a grey dashed arrow. B) Screenshots illustrating the distribution of the RNAPII CRAC signal density around the rRFB. Every track contains overlapped signals to compare either RNAPII CRAC levels in WT (grey) and sen1-3 cells (aqua green) or TES-seq and TSS-seq signals for WT (grey) and rrp6 $\Delta$ (red) as indicated. The TSS and the TES signals of the RNA produced by the fork-trailing transcription (first two tracks) are only visible for rrp6 $\Delta$ cells because the RNA produced is degraded by the exosome and poorly detected in WT cells. Asyn: asynchronously growing cells; $\mathrm{G}_{1}$ : cells arrested in $\mathrm{G}_{1} ; \mathrm{S}_{30 \mathrm{~min}}, \mathrm{~S}_{45 \mathrm{~min}}$ : cells synchronously released in S-phase for $30 \mathrm{~min}$ and $45 \mathrm{~min}$ respectively. At the bottom of the panel, a cartoon illustrates the likely relative position of RNAPII and the fork. 
Monitoring RNAPII by CRAC in the rRFB region in asynchronous cells revealed the existence of a non-annotated transcription unit located upstream and in close proximity of the rRFB, which generates a cryptic unstable transcript that can only be detected in an exosomedefective, $\operatorname{rrp} 6 \Delta$ background as revealed by mapping its 5'- and 3'-ends (TSS and TES, respectively) (Figure 3B). Transcription in this region might generate a co-directional conflict with forks stalled at the rRFB and be of interest for our analysis.

Interestingly, the RNAPII CRAC signal was close to background in $\mathrm{G}_{1}$-arrested cells yet was markedly visible both at the early (30 min release in S-phase) and late (45 min release) replication time points (Figure 3B), indicating that accumulation of RNAPII only occurs during or after replication, possibly as a consequence of fork passage or stalling.

Most importantly, the RNAPII CRAC signal was found to be considerably increased in sen1-3 cells indicating that the interaction of Sen 1 with the replisome is required for releasing RNAPII at this site while in close proximity with a replication fork. Increased RNAPII signal in sen1-3 cells was not due to increased rDNA copy number in this strain as verified by qPCR (data not shown). Analysis of published ChIP-exo data (Rossi et al., 2021) confirmed the specific presence of Sen1 at the DNA in close correspondence with the RNAPII CRAC peak (Figure S4A). The RNAPII peak is located roughly $100 \mathrm{nt}$ from the edge of the rRFB, which is hardly compatible with Fob1 roadblocking RNAPII as we have previously shown that the elongation complex stalls much closer (-10 to $-15 \mathrm{nt}$ ) to DNA bound factors (Candelli et al., 2018; Colin et al., 2014). Rather, the position of the RNAPII peak is compatible with one co-directional replication fork derived from the closest ARS filling the gap between the RNA polymerase and the Fob1-bound rRFB (see scheme in Figure 3B). We cannot exclude that a head-on conflict occurs with the fork originating from the distal ARS and progressing in the opposite direction relative to transcription (Figure $3 \mathrm{~A}$ ), although this would imply distal fork stalling for undetermined reasons after crossing the rRFB.

Together these data provide evidence for replication-dependent RNAPII accumulation, most likely in the wake of the replication fork stalled at the rRFB. Importantly, they also demonstrate 
that the interaction of Sen1 with the replisome is required for the efficient release of RNAPIIs paused in close proximity with the replisome.

\section{R-loops are detected at the rRFB by H-CRAC}

Several studies have linked mutation or depletion of Sen1 to the accumulation of Rloops at sites of conflicts, which has been proposed to generate genomic instability. However, as highlighted above, accumulation of R-loops in the absence of general Sen1 functions might depend on or be exacerbated by major alterations in the transcriptional load that challenges replication. The requirement of $\mathrm{RNase} \mathrm{H}$ activity for the viability of sen1-3 cells suggests that degradation of R-loops is essential in at least some genomic locations when Sen1 cannot interact with the replisome. Therefore, we decided to generate genome-wide maps of R-loops taking advantage of the unprecedented benefits offered by the sen1-3 mutant.

Currently available tools to produce genome-wide R-loops maps have limited resolution and often lack directionally. These techniques rely on immunoprecipitation of DNA:RNA hybrids by the S9.6 antibody after nucleic acid extraction (DRIP-seq and related techniques), or the monitoring of catalytically-dead RNase $\mathrm{H} 1$ occupancy by chromatin immunoprecipitation (R-ChIP) (for a review see Chédin et al., 2021). These approaches do not always provide consistent outputs (Chédin et al., 2021) and we also feared that their resolution would not be sufficient for an integration with our RNAPII CRAC data. Therefore, we devised an alternative method: we reasoned that since $\mathrm{RNase} H$ binds and degrades the RNA moiety of DNA:RNA heteroduplexes, it should be possible to catch it in action at its targets in vivo by UV-crosslinking. Purification of the enzyme under denaturing conditions as in CRAC should allow sequencing of the crosslinked RNA for a sensitive and high-resolution detection of the hybrids. A similar strategy was successfully used to detect exosome targets (Delan-Forino et al., 2017), and should provide in vivo data obviating alterations in R-loop metabolism that might occur by expressing RNase $\mathrm{H} 1$ catalytic mutants. H-CRAC (for RNase $\underline{H}-\underline{\text { CRAC) }}$ experiments performed with both RNase $\mathrm{H} 1$ and RNase $\mathrm{H} 2$ provided very reproducible and similar outputs, despite revealing some specificities (Figure 4A and S3A, see 
bioRxiv preprint doi: https://doi.org/10.1101/2022.02.09.479708; this version posted February 10, 2022. The copyright holder for this preprint (which was not certified by peer review) is the author/funder, who has granted bioRxiv a license to display the preprint in perpetuity. It is made available under aCC-BY-NC-ND 4.0 International license.

below). This was expected in the light of the known redundancy of these enzymes in R-loop degradation.

Figure 4

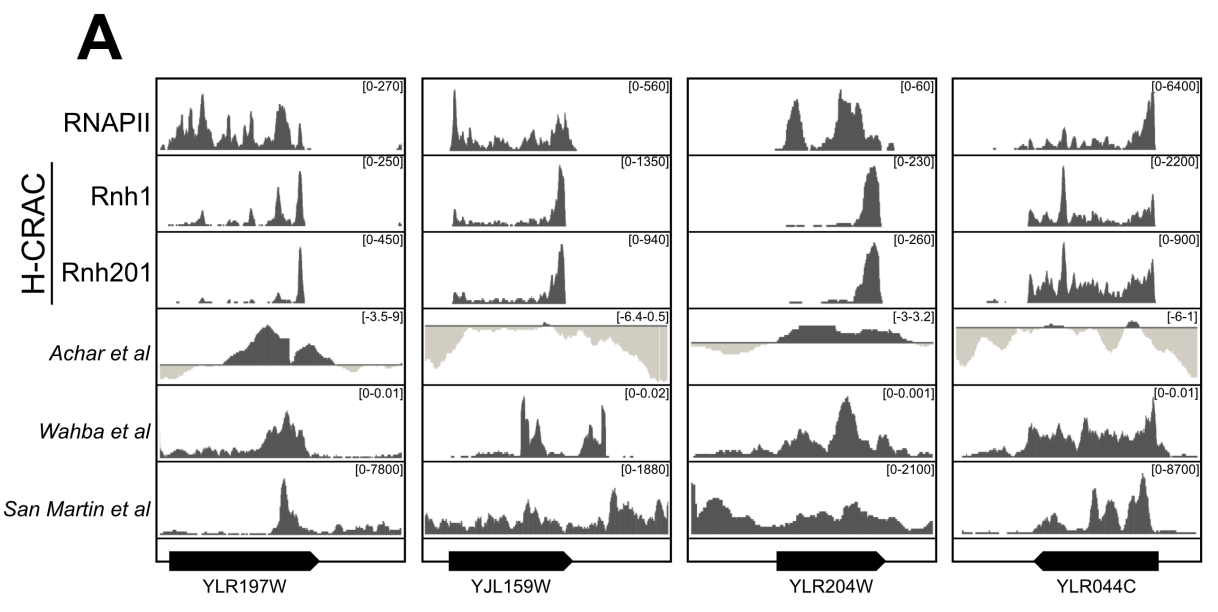

B

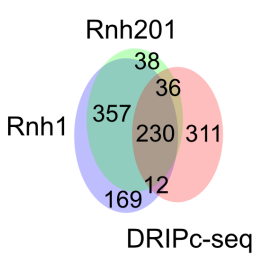

Fisher's exact test:

Rnh1 vs Rnh201 $\cong 0$

Rnh1 vs DRIPc-seq $=2.3 \mathrm{e}-67$

Rnh201 vs DRIPc-seq $=4.2 \mathrm{e}-105$
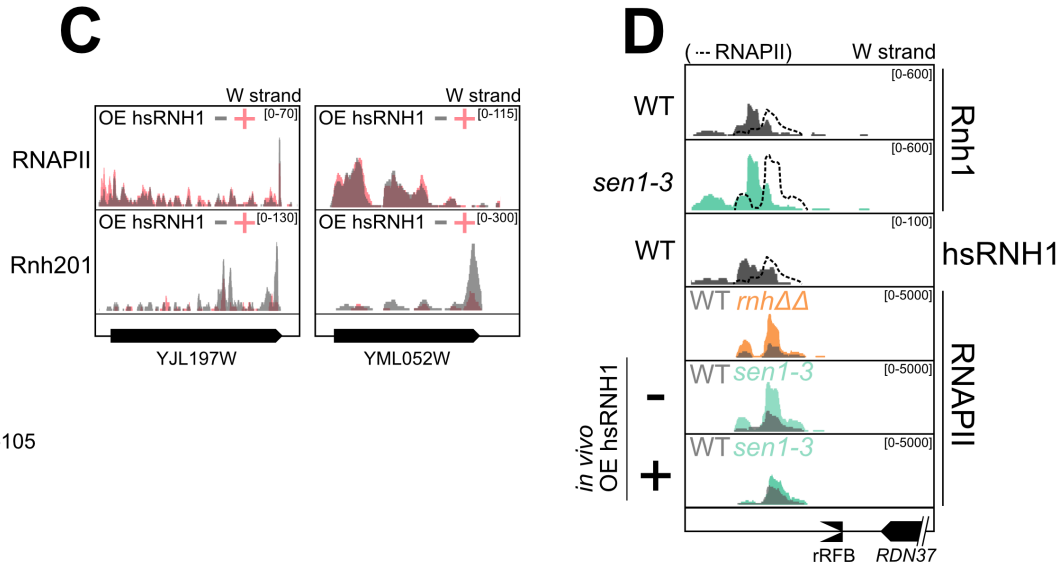

\section{Figure 4: RNase H partakes in Sen1-dependent RNAPII release at the RFB}

A) Snapshots of individual genes illustrating the comparison between DRIP datasets (Achar et al., 2020; San Martin-Alonso et al., 2021; Wahba et al., 2016) and H-CRAC signals obtained from Rnh1 and Rnh201. RNAPII CRAC is also shown for evaluating the R-loop signals relative to transcription. For the directional DRIP-seq (San Martin-Alonso et al., 2021), the H-CRAC and the RNAPII CRAC only the strand of the target gene is shown. B) Overlap of the genes containing the highest signals (levels higher than the mean plus one standard deviation) defined by the directional DRIP-seq (San Martin-Alonso et al., 2021) and by H-CRAC (Rnh1 or Rnh201). The significance of the overlap between H-CRAC and the DRIP-seq dataset was calculated with Fisher's exact tests and is indicated at the bottom. 
C) Representative examples of decrease in H-CRAC (Rnh201) signals upon in vivo overexpression of hsRNH1. RNAPII occupancy by CRAC in both conditions is also shown to underscore that reduction of H-CRAC signal is not due to decreased transcription. D) H-CRAC and RNAPII CRAC at the rRFB in different genetic backgrounds as indicated: R-loops accumulation at the rRFB as detected by Rnh1 and hsRNH1 H-CRAC. The position where RNAPII accumulates in the corresponding background is indicated by a dashed curve. Increased RNAPII occupancy is observed at the rRFB by RNAPII CRAC in $r n h 1 \Delta r n h 201 \Delta(r n h \Delta \Delta)$ cells. RNAPII increased occupancy is suppressed by in vivo overexpression of hsRNH1.

To assess the reliability of our approach, we gauged the validity of the proposed landmarks for R-loop detection (Chédin et al., 2021). We first ectopically expressed in yeast a sequence derived from the mouse AIRN gene that was demonstrated to form R-loops in vitro and in vivo (Carrasco-Salas et al., 2019; Ginno et al., 2012). We verified that this sequence, when transcribed in S. cerevisiae, generates high levels of RNase H-sensitive Rloops, as determined by DRIP followed by quantitative PCR (Figure S3B). Prominent H-CRAC signals were detected at the ectopically expressed mAIRN locus (Figure S3C), validating the notion that $\mathrm{H}$-CRAC robustly identifies well-established regions of R-loop formation.

$\mathrm{H}$-CRAC signals overlapped transcription at the genome-wide scale, as expected considering the co-transcriptional nature of R-loop formation (Figures 4A, S3D and S3E), but the pattern of the RNAPII and RNase H signals was generally different (Figures 4A and S3E), consistent with the notion that not all transcribed regions generate R-loops to the same extent.

$\mathrm{H}-\mathrm{CRAC}$ signals with both RNase $\mathrm{H} 1$ and RNase $\mathrm{H} 2$ were markedly strand specific (Figure S3E), with distinct and well-defined peaks (Figure 4A). A comparison with the only directional R-loop map generated by S9.6-DRIP-seq in yeast (San Martin-Alonso et al., 2021) revealed significant overlaps when taking into account the overall signal along coding genes (Figures 4A, 4B). However, the resolution of the signal was clearly higher and its distribution within genes often different, with prominent $\mathrm{H}-\mathrm{CRAC}$ signals observed at new locations (Figure 4A and data not shown). The detailed genome-wide analysis of R-loops distribution determined by H-CRAC is beyond the scope of this report and will be provided in a separate manuscript (Aiello et al., in preparation). 
Sensitivity of signals to overexpression of human RNase $\mathrm{H}$ is a landmark to gauge the reliability of R-loop detection (Chédin et al., 2021). Therefore, we overexpressed human $\mathrm{RNH} 1$ in cells that also expressed a version of Rnh201 suitably tagged for H-CRAC, and analysed the data after normalization to an S. pombe spike in. Rnh201 H-CRAC signals were considerably reduced in many locations (Figure 4C), without a significant, general effect on transcription (Figure 4C and S3F), which was monitored in parallel to ascertain that reduced H-CRAC signals were not due to altered gene expression. This indicates that although ectopically expressed hsRNH1 cannot fully outcompete tagged endogenous Rnh201, it can significantly reduce R-loop levels. Consistently, we could also generate similar H-CRAC signal distributions using tagged hsRNH1, confirming that the ectopically expressed, heterologous enzyme recognizes very similar targets as the yeast proteins (Figures S3G and $\mathrm{S} 3 \mathrm{H}$ ). Together, these data demonstrate that H-CRAC is a sensitive and resolutive method for detecting in vivo at least a significant fraction of cellular DNA:RNA hybrids, overlapping and complementing in vitro DRIP-based methods. Similar to DRIP, H-CRAC cannot distinguish between DNA:RNA hybrids and R-loops, the latter being defined by the presence of a single stranded DNA portion. However, known non-R-loop DNA:RNA hybrids would either be too short (e.g., Okazaki fragments) or protected from RNase $\mathrm{H}$ crosslinking (e.g., the hybrid contained in the RNAPII inner channel) to be detected by H-CRAC. With this caveat in mind, we will therefore use preferentially the terms R-loops or RNase $\mathrm{H}$ targets for the structures generating H-CRAC signals.

To assess whether R-loops form at the rRFB during replication, we generated $\mathrm{H}$-CRAC maps in sen1-3 and wild-type cells. Interestingly, we detected prominent Rnh1 H-CRAC signals peaking roughly $50 \mathrm{nt}$ upstream of the fork-trailing RNAPII peak (Figures 3B and 4D), consistent with the notion that they form immediately upstream of the stalled polymerase. Rnh201 signals were less prominent in this position and were instead preferentially observed roughly 500 nt upstream (Figure S4A), suggesting that Rnh1 might preferentially recognize Rloops at this site. Ectopically-expressed human RNase $\mathrm{H}$ (hsRNH1) for H-CRAC generated 
clear signals that nicely overlapped yeast Rnh1 targets in this region (Figure 4D), further supporting the notion that they represent bona fide R-loops.

H-CRAC signals proximal to the rRFB increased significantly in sen1-3 cells in their absolute levels but not when evaluated relative to the levels of paused RNAPII upstream of the replication fork (Figure 4D). This finding indicates that higher R-loop levels at this site parallel the increased stalling of RNAPII engaged in conflicts with replication forks when Sen1 cannot interact with the replisome.

From these experiments we conclude that R-loops form upstream of RNAPII conflicting with the stalled replication forks. The interaction of Sen1 with the replisome is required for releasing fork-trailing RNA polymerases, but not for limiting the levels of R-loops that form per transcriptional event.

\section{RNases H promote RNAPII release at the rRFB}

The strong growth defect of sen1-3 cells in the absence of RNase $\mathrm{H}$ activity, suggests that Sen 1 at the replisome and RNases $\mathrm{H}$ have a common or complementary function either in limiting R-loops accumulation, in RNAPII release, or both. Because we did not observe increased R-loops per transcription event at the rRFB in sen1-3 cells, we considered a possible implication of RNases H/R-loops in RNAPII release. We first monitored RNAPII

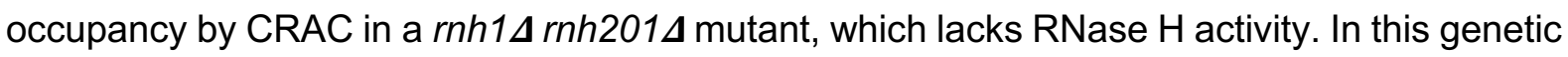
context, transcription was not generally altered, as shown by the profiles of median RNAPII CRAC signals on genes (Figure S4B). Interestingly, however, we observed a clear increase in the levels of RNAPII at the rRFB compared to a wild-type strain, which accumulated in the same position and to similar levels as in sen 1-3 cells (Figure 4D). Thus, RNases $\mathrm{H}$ are required to limit accumulation of RNAPII at the TRC in the rRFB.

RNases $\mathrm{H}$ might contribute to the release of the polymerase independently of R-loop degradation, or by degrading the RNA moiety of these structures. In this latter perspective the expected increase in R-loops in $r n h 1 \Delta r n h 201 \Delta$, might prevent efficient RNAPII release. One important prediction of this hypothesis is that degrading the R-loops formed upstream of the 
stalled polymerase in sen1-3 cells should favour its release and suppress the RNAPII accumulation phenotype.

Overexpression of human hsRNH1 significantly reduced R-loop levels genome-wide, without generally altering transcription, as determined by RNAPII CRAC analyses (Figures 4C and S3F), yet this was accompanied by a significant reduction in RNAPII accumulation at the rRFB in sen1-3, to similar levels as in wild-type cells (Figure 4D). Together, these results suggest that modulating the levels of R-loops at the rRFB, either by increasing them in $r n h 1 \Delta$ rnh2014 cells or by decreasing them upon overexpression of hsRNH1, affects RNAPII release in an anti-correlative manner.

In the light of these results, we considered that RNases $\mathrm{H}$ might also contribute to the release of RNAPII that stall at TRCs in the 5'-end of genes undergoing replication. In this perspective it is expected that the absence of RNase $\mathrm{H}$ activity should generate a 5'-skewed RNAPII pattern similar to the one observed in sen1-3 cells in asynchronous cells (Figure 1A). However, the double rnh14 rnh2014 mutant did not phenocopy the sen1-3 RNAPII 5'-end accumulation suggesting that RNases $\mathrm{H}$ do not partake in releasing RNAPII in these locations (Figure S4B). This conclusion is also supported by the findings that overexpression of hsRNH1 in sen1-3 cells did not suppress 5'-end RNAPII accumulation (Figure S4C, compare with Figure 2A) and that R-loops are not significantly detected in the very 5'-end of genes where the increased RNAPII accumulation is observed (Figures S4D and S4E). One likely explanation for these results is that in the proximity of the TSS the short length of the available nascent RNA is not compatible with the formation of DNA:RNA hybrids (see Discussion).

From these experiments we conclude that RNAPII is released at the rRFB by the combined action of Sen1 at the replisome and RNase $\mathrm{H}$, which presumably exerts its function by degrading the R-loops formed in the wake of the stalled transcription elongation complex.

\section{Sen1 releases RNAPII at sites of conflicts with RNAPIII}

Aside from RNAPII and replisome components, Sen1 also interacts with RNAPIII, an interaction that is also lost in sen1-3 cells (Xie et al., 2021). We have shown that this latter 
interaction is not mediated by the replisome but reflects the existence of an alternative complex since quantitative MS analysis of the RNAPIII interactome detected Sen1 but not replisome components.

We have previously shown that non-coding and non-annotated RNAPII transcription events might collide with RNAPIII transcription units, where they are restrained by roadblocks located in the 5'- and 3'-end of tRNA genes (Candelli et al., 2018). The mechanism by which RNAPII is released at these sites was not addressed, although we showed that RNAPII ubiquitylation and degradation occurred at other sites of roadblock. We hypothesized that, analogous to the role at the replisome, interaction with RNAPIII might recruit Sen1 for removing conflicting RNAPIls. Therefore, we monitored RNAPII transcription around tRNA genes in wild-type and sen1-3 cells. RNAPII was found to accumulate upstream of tRNA genes in sen1-3 cells, consistent with the notion that conflicting RNAPII is not released efficiently when Sen1 cannot interact with RNAPIII. This can be clearly appreciated at individual cases (Figure 5A), and more generally by the heatmap analysis of the whole tRNA genes population (Figure 5B). We considered the possibility that this accumulation was related to ongoing replication, but the same analysis performed in $\mathrm{G}_{1}$-arrested cells revealed very similar effects (Figures 5A and 5B). Increased accumulation of RNAPII was also observed when monitoring antisense transcription relative to tRNA genes (Figures $5 \mathrm{~A}$ and $5 \mathrm{~B}$, right panels), although to lower levels, possibly because additional mechanisms are in place for limiting head-on conflicts. Nevertheless, we clearly observed that, when uncontrolled by Sen1, RNAPII entered tRNA transcription units in the antisense direction (for technical reasons we cannot monitor sense RNAPII transcription in the body of tRNA genes). Similar to what observed at mRNA coding genes, no significant effect was observed after deletion of the two RNase $\mathrm{H}$ genes (Figure S5A), strongly suggesting that degradation of R-loops is not playing an important role at these sites.

From these observations, we conclude that Sen1 plays important roles in limiting conflicts between RNAPII and RNAPIII at tRNA genes, a role that is dependent on its interaction with RNAPIII but is independent from ongoing replication. 


\section{Figure 5}
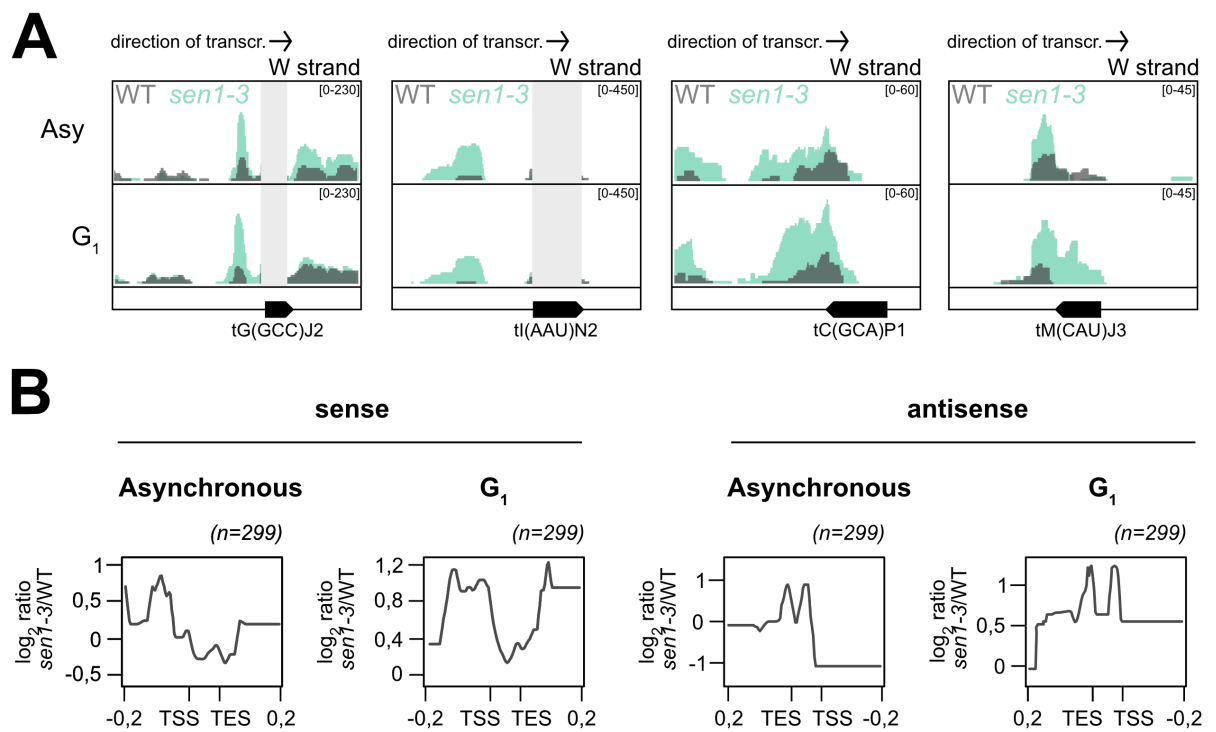

sense

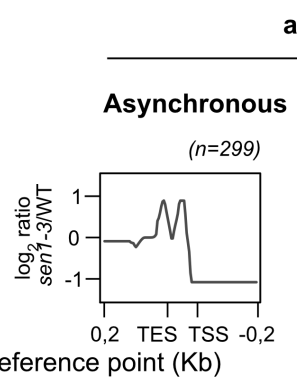

antisense
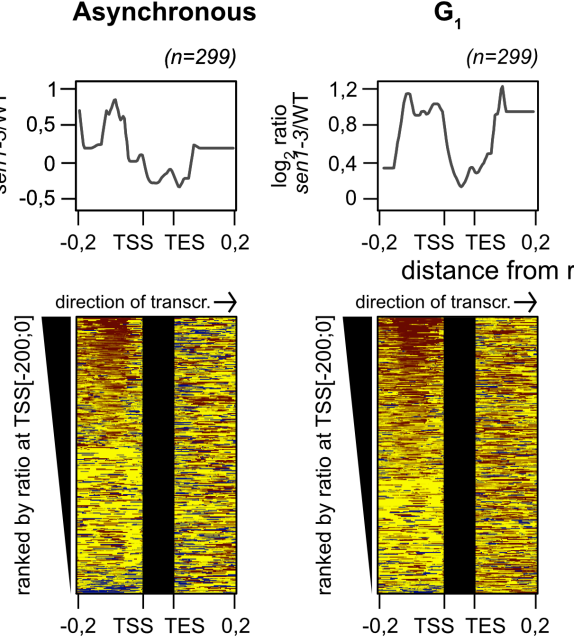

distance from reference point $(\mathrm{Kb})$
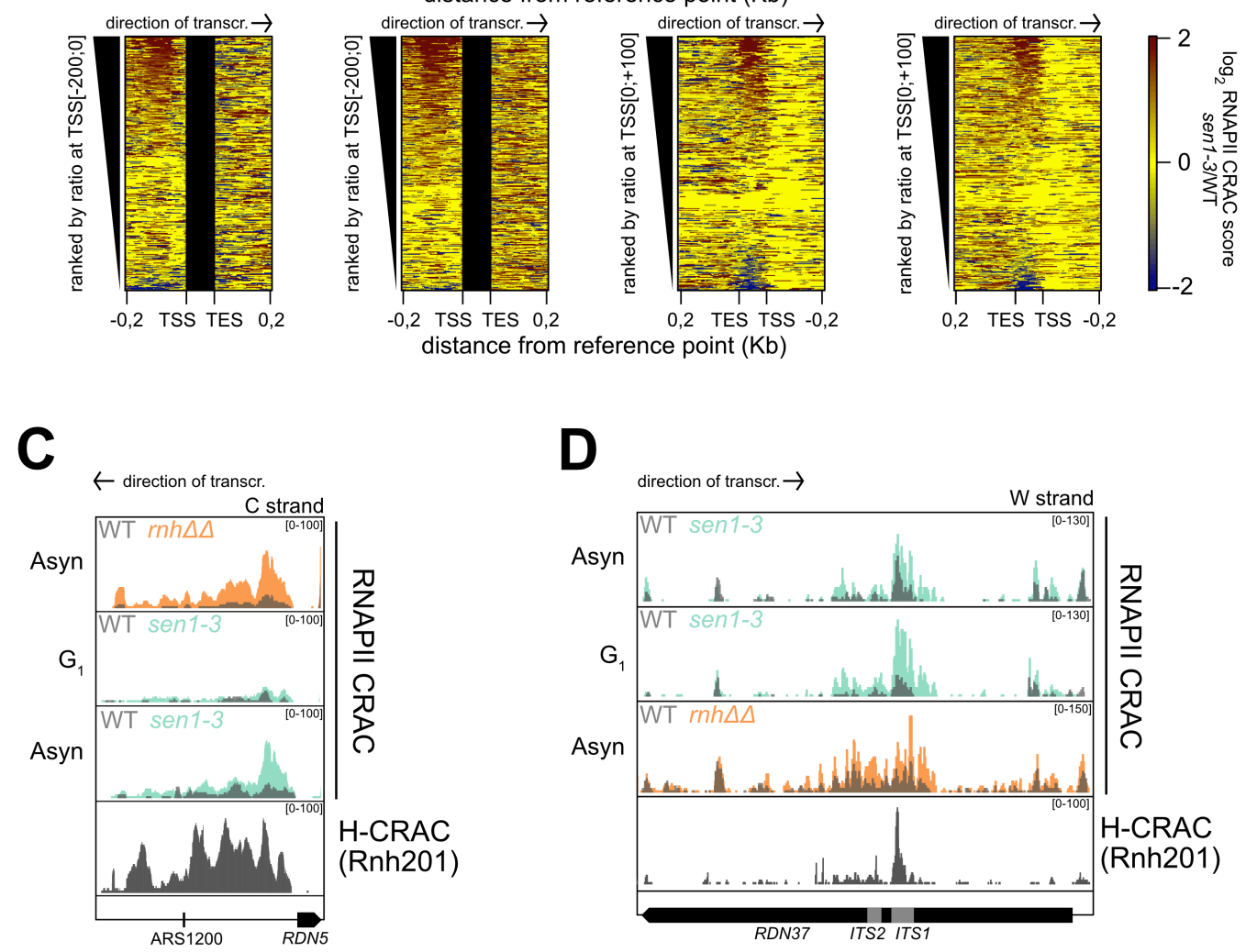

Figure 5: Sen1 releases RNAPII at sites of conflicts with RNAPIII and limits RNAPII transcription in the ribosomal DNA 
A) RNAPII accumulation in the sense (left panels) or antisense (right panels) orientation at individual, representative tRNA genes in sen1-3 cells. WT and sen1-3 signals have been overlapped for easy comparison as in Figure 2. The strand of the tRNA gene and the direction of RNAPII transcription are indicated. Sense RNAPII signals within the tRNA body are masked because they cannot be reliably distinguished from contaminating mature tRNAs. Asyn: asynchronously growing cells; $\mathrm{G}_{1}$ : cells arrested in $\mathrm{G}_{1}$ with $\alpha$-factor.

B) Heatmap analyses representing the $\log _{2}$ ratio of the RNAPII signal in sen1-3 vs WT cells at tRNA genes aligned on their TSS and TES respectively for the sense (left panels) and the antisense transcription (right panel). For RNAPII transcription that is sense relative to the tRNAs, genes were ranked by the $\log _{2}$ ratio in the 100 nt window preceding the TSS, which is where the RNAPII peak is generally observed. As in A, signals within the tRNA body are masked. For antisense transcription, it is possible to monitor antisense RNAPII signals within the body of the tRNA gene, which is where the ratio sen1-3/WT increases the most. In these heatmaps, ranking was done based on this region. The summary plot on the top was calculated using the median values for each position.

C) RNAPII occupancy antisense and upstream of the RDN5 gene, in sen1-3 and RNases H deleted cells $(r n h \Delta \Delta)$. $\mathrm{H}-\mathrm{CRAC}$ signal for Rnh201 is also shown for the same region. The $\mathrm{C}$ strand is monitored (antisense of RDN5 transcription) and the direction of RNAPII transcription is indicated. D) CRAC RNAPII occupancy antisense to the RDN37 gene in WT, sen1-3 and RNases $\mathrm{H}$ deleted cells $(r n h \Delta \Delta)$. R-loops detection by Rnh201 H-CRAC is also shown.

\section{Roles of Sen1 and RNases $H$ in limiting RNAPII transcription in the ribosomal DNA}

Although the ribosomal loci are mainly devoted to the production of rRNA by RNAPI and RNAPIII, transcription by RNAPII is also known to occur, mainly antisense to the transcription unit producing the $37 \mathrm{~S}$ rRNA precursor. Because the $5 \mathrm{~S}$ rRNA is produced by RNAPIII, we first investigated whether the sen1-3 mutation would affect RNAPII occupancy around the RDN5 gene. Akin to other RNAPIII transcription units, we clearly observed replicationindependent (i.e., observed also in $\mathrm{G}_{1}$-arrested cells) RNAPII accumulation antisense of $R D N 5$ in sen1-3 cells (Figure S5B). RNAPII accumulation was also observed in $r n h 1 \Delta$ rnh2014 cells suggesting that RNase $\mathrm{H}$ is required at this locus for efficient RNAPII release. Consistently, R-loops were detected, both by H-CRAC and DRIP (San Martin-Alonso et al., 2021; Wahba et al., 2016) immediately upstream of the paused polymerase (Figure S5B).

Interestingly, we also noticed the occurrence of transcription upstream and antisense of RDN5 directed towards the close rARS (ARS1200) replication origin (Figure 5C), which was 
only observed in replicating cells and was also RNases $\mathrm{H}$ dependent. R-loops were detected by $\mathrm{H}-\mathrm{CRAC}$ overlapping the whole region of transcription separating the replication origin from the RDN5 gene, most likely landmarking sites of head-on transcription-replication conflicts. Thus, around RDN5 Sen1 has replication-dependent and -independent roles in limiting conflicts involving RNAPII transcription.

These findings prompted a closer examination of RNAPII transcription in the rDNA

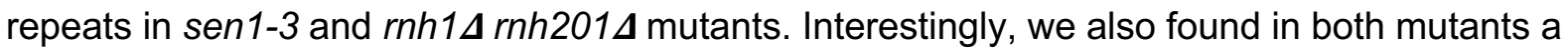
region of increased RNAPII occupancy antisense of RDN37 transcription, roughly corresponding to the internal transcribed spacer 1 (ITS1, Figure 5D). This increase was not dependent on replication as it was observed in $\mathrm{G}_{1}$-arrested cells and was associated to the formation of R-loops detected with H-CRAC for both Rnh1 and Rnh2 (see Discussion).

We conclude from these data that Sen1 and RNases $\mathrm{H}$ play important roles in resolving conflicts involving RNAPII transcription in the ribosomal DNA region.

\section{RNase $\mathrm{H}$ activity and the dual roles of Sen1 transcription termination and in conflict-solving are required for maintaining genome stability}

We set up to explore the consequences of the sen1-3 mutation on genomic stability. We first assessed the sensitivity of sen 1-3 cells to replication stress induced by hydroxyurea (HU) and methyl methanesulfonate (MMS). We found a moderate and strong sensitivity to HU and MMS respectively (Figure $6 \mathrm{~A}$ ), indicating that association of Sen1 with the replisome is required for optimal replicative stress response. However, and as we had previously described (Appanah et al., 2020) we did not observe the same genomic instability phenotypes reported for the loss-of-function sen1-1 mutant, both at the level of Transcription Associated Recombination (Mischo et al., 2011) (Figure S6A), and Rad52 foci formation, a hallmark of DSB accumulation and repair (see below, Figure 6D). Also, we did not observe an increase in R-loops by H-CRAC (Figures S4E, S4F and S4G), coherent with previous immunofluorescence analysis of chromosome spreads (Appanah et al., 2020). These differences could be explained if the alterations in the transcription landscape observed in 
Sen1 loss-of-function but not in sen1-3 cells (Figure 1) contribute significantly to the genomic instability phenotypes. Also, the functional cooperation with $\mathrm{RNases} \mathrm{H}$, underlying the strong genetic interaction with sen1-3, might mask phenotypes linked to the loss of interaction with the replisome and RNAPIII. Therefore, we devised a genetic system for analysing the damage induced by the combination of the sen1-3 and rnh14 rnh2014 mutations. We constructed an inducible triple mutant whereby a chromosomal wild-type, AID-tagged Sen1 complements lethality of a rnh14 rnh2014 strain containing a plasmid-borne, untagged sen1-3 allele. Upon addition of auxin (IAA) the wild-type copy is rapidly degraded leaving only the Sen1-3 protein (Mendoza-Ochoa et al., 2019). Induced triple mutant cells only partially recapitulated the lethality of the bona fide triple mutant, possibly because wild-type Sen1 was not fully depleted and/or slight overexpression of sen1-3 partially suppressed its phenotype (Figure S6B). However, combination of the IAA treatment and incubation at the suboptimal temperature of $37^{\circ} \mathrm{C}$ led to a similar level of growth impairment as the one observed for Sen1 depletion. We thus carried our DNA damage analysis also at this temperature. We first monitored the extent of DNA damage by measuring the frequency of Rad52-YFP foci upon induction of the triple mutant phenotype (Figures 6B, S6C and S6D). In the non-induced triple mutant, we observed, as expected, the same Rad52 foci frequency observed in a double $r n h 1 \Delta \mathrm{rnh} 201 \Delta$ deletion. Importantly, partial induction of the triple mutant phenotype led to a significant increase in the number of Rad52-YFP foci compared to the double RNases $\mathrm{H}$ deletion, implying that in this context association of Sen1 with the replisome is required for limiting DNA damage.

Another hallmark of DNA damage, H2A histone phosphorylation at position S129, was monitored by western blot detection. Consistent with the increased Rad52-YFP foci, phosphorylation of $\mathrm{H} 2 \mathrm{~A}$ was found to be significantly increased when the triple mutant, partial phenotype was transiently induced by the addition of IAA (Figures $6 \mathrm{C}$ and S6E).

To assess if the transcription termination defects in Sen1 loss-of-function mutants significantly aggravate the phenotypes linked to the absence of Sen1 at the replisome, we induced a non-coding transcription termination defect in sen1-3 independently of Sen1 by additionally mutating Nrd1, another component of the NNS complex. We reasoned that if the 
Sen1 loss-of-function phenotypes were due to a combined termination and conflict-solving defect, a double nrd1-102 sen1-3 mutant should recapitulate the phenotypes of sen1-1 cells. This expectation was fully met when analysing the cellular frequency of Rad52 foci in the single and double mutants. Foci were poorly detected in single mutants, consistently with previous reports (Appanah et al., 2020; Costantino and Koshland, 2018; Mischo et al., 2011), but were found to similar levels in sen1-1 and $n r d 1-102$ sen1-3 cells (Figure 6D).

From these results we conclude that by interacting with the replisome and RNAPIII, Sen1 plays important roles at sites of transcription-transcription and transcription-replication conflicts. In the rDNA this role is redundantly exerted by RNases $\mathrm{H}$. In the absence of both enzymes or in the presence of transcription termination defects, extensive DNA damage occurs, which is likely underlying lethality.

\section{Figure 6}
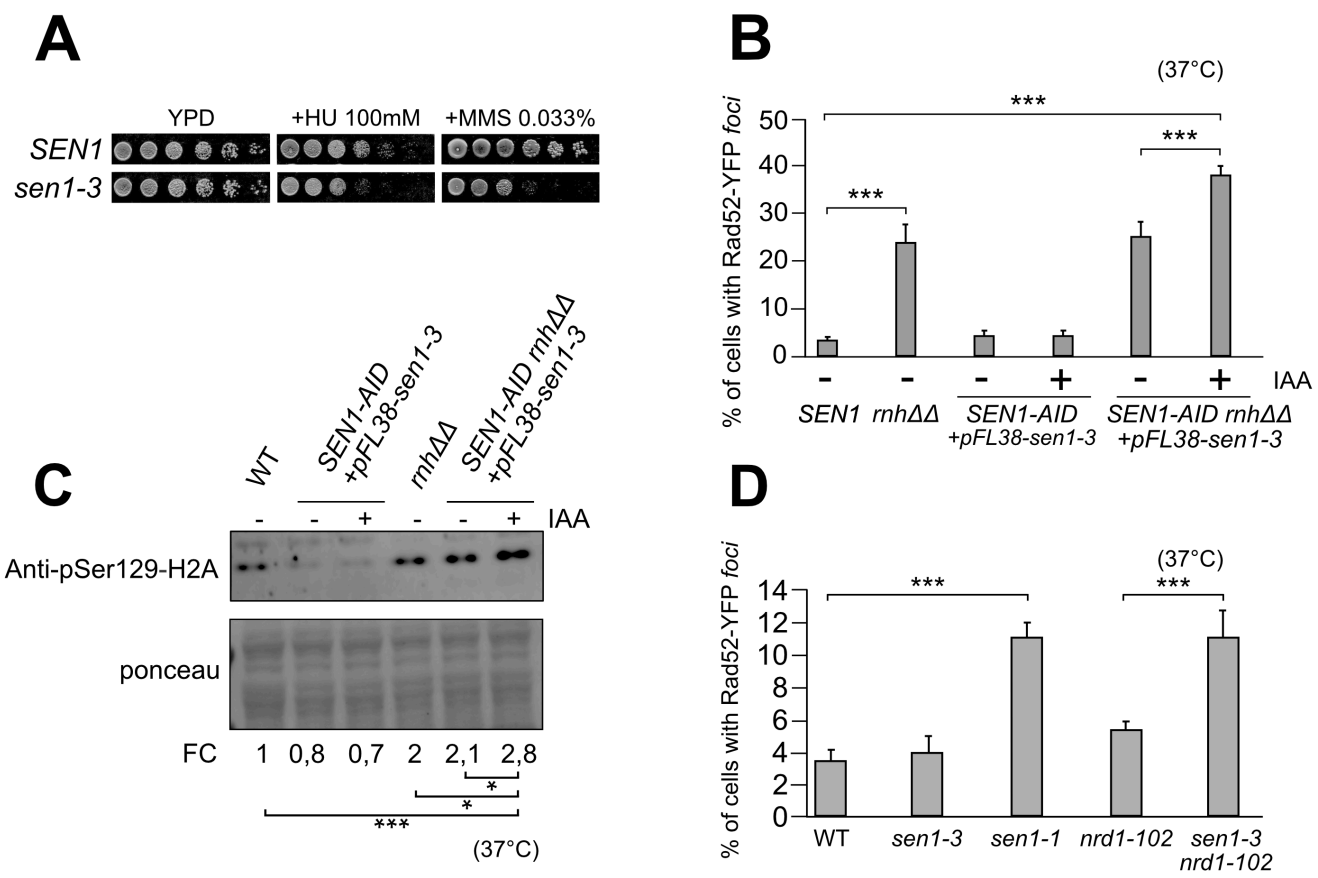

Figure 6: Sen1 cooperates with RNases $\mathrm{H}$ to maintain genome stability

A) Growth assay of sen1-3 and WT cells as a control in the presence or absence of hydroxyurea (HU) and methyl methanesulfonate (MMS) as indicated. Plates were incubated for 3 days at $30^{\circ} \mathrm{C}$. Growth assays were performed 
bioRxiv preprint doi: https://doi.org/10.1101/2022.02.09.479708; this version posted February 10, 2022. The copyright holder for this

preprint (which was not certified by peer review) is the author/funder, who has granted bioRxiv a license to display the preprint in perpetuity. It is made available under aCC-BY-NC-ND 4.0 International license.

on the same plates for each series (i.e., HU or MMS). B) Frequency of cells containing Rad52-YFP foci in asynchronously growing cultures incubated at $37^{\circ} \mathrm{C}$ for $1 \mathrm{~h}$ for the indicated strains, in presence or absence of auxin (IAA) to deplete WT Sen1-AID. ${ }^{* * *} p<0.001$. Standard deviation $(n=3)$ is indicated. C) Western blot detection of $\mathrm{H} 2 \mathrm{~A}$ Ser129 phosphorylation in asynchronously growing cultures incubated at $37^{\circ} \mathrm{C}$ for $1 \mathrm{~h}$ for the indicated strains, in presence or absence of auxin (IAA) to deplete WT Sen1-AID. The average level quantified from three independent replicate (Figure S6C) is shown at the bottom. ${ }^{*} p<0.5 ;{ }^{* *} p<0.001$. D) Rad52-YFP foci are monitored in the indicated strains as in Figure 6B. Coupling of the sen1-3 allele to Sen1-independent transcription termination defects generated by the nrd1-102 leads to levels of DNA damage comparable to the ones observed in sen1-1 cells. ${ }^{* *} p<0.001$. Standard deviation $(n=3)$ is indicated. 


\section{DISCUSSION}

Synchronous occupancy of the compact yeast genome by several cellular machineries that exert essential functions in the expression, maintenance and transmission of the genetic information requires the existence of robust mechanisms that ensure the coordination of the different processes, prevent conflicts, or solve them when they occur. Dealing with the extensive occupancy of the genome by transcription events that largely overcome the limits of functional gene annotations is a major challenge in this context. In this study we employed high resolution and innovative genomic tools to elucidate the functions of the Sen1 helicase and RNases H in "genomic distancing". Importantly, and as opposed to previous reports (Alzu et al., 2012; Costantino and Koshland, 2018; Mischo et al., 2011; Zardoni et al., 2021), this study was performed without altering the physiological transcriptional landscape and in the absence of global replication stress. We propose a model that implicates these enzymes in many sites to control transcription-replication and transcription-transcription conflicts. Importantly, we revisit the role of Sen1 in genomic stability and transcriptional homeostasis by disentangling these functions, whose synthetic association likely impinges on the many phenotypes previously described.

\section{Loss of Sen1 function generates termination and conflict-solving defects that}

\section{conjunctly lead to genomic instability}

Many earlier studies, including from our laboratory, have clearly established a role for Sen1 in transcription termination of several thousands of non-coding RNA genes (for a review see Porrua et al., 2016). The biochemical mechanism of Sen1-dependent termination has also been extensively studied in vitro (Porrua and Libri, 2013; Han et al., 2017; Leonaitè et al., 2017; Wang et al., 2019). Genome-wide analyses of the effects of Sen1 depletion (this study; see also Schaughency et al., 2014) demonstrate the inductions of major alterations in the transcriptional landscape. These alterations in the transcription scenery have a large potential for increased interference with concurrent processes, together with global alterations in gene expression homeostasis and cellular physiology. Indeed, we also describe changes in the 
gene expression program, which, at least to some extent, parallel the ribosome assembly stress described recently by the Shore and Churchman laboratories (Tye et al., 2019; Zencir et al., 2020). These phenotypes are possibly triggered by defective production of snoRNAs, which are major NNS targets and contribute to rRNA maturation, or by defects in transcription termination of rRNA genes, a process in which Sen1 has been implicated (Kawauchi et al., 2008). Together, these results demonstrate that the transcription and gene expression program of Sen1 loss-of-function mutants is largely distinct from the one of wild-type cells, and raise questions about the assessment of genomic instability phenotypes in such nonphysiological conditions.

It has been shown that alterations in transcription termination generated with other NNS mutants (i.e., nrd1 and nab3) do not induce instability per se (Alzu et al., 2012; Costantino and Koshland, 2018; Mischo et al., 2011). These earlier findings indicate that transcription termination defects are not sufficient, alone, to generate genomic instability, most likely because control or prevention of conflicts rely on robust and redundant mechanisms. Still, direct or indirect effects of impaired termination can generate instability when coupled to other defects due to altered Sen1 functions. Fully consistent with this notion is our finding that impairing transcription termination independently of Sen1 (i.e., by mutation of Nrd1) in sen1-3 cells, fully recapitulates the DNA damage phenotype of sen1-1 cells, which are defective for both termination and conflict-solving functions of Sen1 (Figure 6). The simplest interpretation of these results is that when termination alone is impaired, the increased transcriptional challenge at sites of conflicts can still be resolved by Sen 1 and RNases $H$, while in the additional absence of this conflict-resolving function increased DNA damage occurs or is not repaired efficiently.

\section{On the physiological relevance of Sen1 binding to the replisome}

Focal to our study is the sen1-3 mutant, which we have previously shown to fully lose interaction with replisome components (Appanah et al., 2020) and with RNAPIII (Xie et al., 2021). Our working hypothesis underlying this study has been that the interaction of Sen 1 with 
the replisome allows either its recruitment or its function at sites where transcription collides with replication to dismantle the elongation complex for giving way to replication. We have shown that sen 1-3 cells have no transcription termination defects or altered gene expression, which allowed studying the role of Sen1 at sites of conflicts under overall physiological conditions in terms of transcription. Because sen1-3 cells have no major growth or hyperrecombination phenotypes (Figures 6 and S6), it would be legitimate to question the physiological relevance of the functions impaired in sen1-3 cells. However, several observations support the notion that the sen 1-3 mutation affects important facets of Sen1 function: i) viability is not supported in the absence of RNase $\mathrm{H}$ activity (Figure S6B); ii) growth is affected in the presence of genotoxic agents (Figure 6A); iii) the speed of the replication forks is decreased (Figure S2H); iv) DNA damage levels increase in a rnh14 rnh2014 background or when transcription termination is additionally affected (Figures 6B and 6D); v) altered RNAPII distribution is detected in several locations where TRCs are expected to occur (Figure 2); vi) defective RNAPII release occurs at sites of conflicts with RNAPIII (Figure 5). These findings underscore the notion that redundant mechanisms are in place to back up Sen1 action and/or that major effects can only be observed under challenging conditions. In this perspective, while this work was in progress it was reported that DNA damage and fork stalling occur under Sen1 depletion at sites of TRCs specifically under conditions of HUinduced replication stress (Zardoni et al., 2021). In this study, transcription of genes that host TRCs was also found to be altered, a phenotype that we did not observe even upon Sen1 depletion (data not shown). Our interpretation for these differences is that failure to complete transcription at some genes hosting TRC sites results from a synergistic effect of decreased replication fork progression due to the $\mathrm{HU}$ treatment and the failure to efficiently remove RNAPII by Sen1 at TRCs, which together delays the resolution of conflicts and affect the resumption of novel transcription cycles.

We provide evidence that Sen1, by binding to the replisome, releases RNAPII engaged in conflicts with replication in the very 5'-end of genes, which is the region with the highest average RNAPII persistence in yeast. We also found increased sen1-3-dependent RNAPII 
accumulation in other regions of pausing (Figure 2B) suggesting that it is RNAPII pausing and not the 5'-end of genes per se, that determines the preferential sites of conflicts. A similar, genic RNAPII accumulation was described in dicer mutants in the 3'-end of S. pombe genes, which was also proposed to be due to the defective resolution of TRCs (Castel et al., 2014). The reasons for these differences in the position where RNAPII accumulates are not clear, but they might be due to a different mechanism of action of Sen 1 and Dicer or to the different distribution of RNAPII pausing at $S$. pombe genes.

While this work was in preparation, a similar increase in RNAPII occupancy was reported to occur in cells expressing loss-of-function mutants of the human Sen1 homologue SETX or in $\triangle S E T X$ cells (Kanagaraj et al., 2022). Although it was not shown whether these phenotypes are due to TRCs, they were found more prominently in very long (>100kb) genes, which generally host common fragile sites (CFS). Genes hosting CFS were also shown in this study to be frequently subject to genomic rearrangements in the absence of SETX. Thus, the role of Sen1 in solving TRCs within genes might be conserved for human SETX, and could also be independent from its function in terminating non-coding transcription, which is not clearly established in human cells.

\section{On the relationship between Sen1 and R-loops}

A salient question, central to this study, concerns the functional relationships between Sen1 and RNases $\mathrm{H}$. Although both classes of enzymes have been involved in the resolution of R-loops and have been proposed to work together at these structures (Costantino and Koshland, 2018), we could not globally detect increased R-loop levels in sen1-3 cells by HCRAC (Figures S4E-G), chromosome spreads (Appanah et al., 2020), or by DRIP qPCR at a few R-loop-prone sites (data not shown). This indicates that the interaction of Sen1 with the replisome is not required for suppressing $R$-loops and might suggest that the $R$-loop increase detected in sen1 lack-of-function backgrounds is also linked to the transcriptional termination defect of this mutant background. Because R-loops are known to be sites prone to DNA damage, this notion is consistent with the finding reported here that increased sites of DNA 
damage are observed when associating a termination defect generated by the nrd1-102 allele to the sen1-3 mutation. In this perspective, it is possible that Sen1, rather than unwinding Rloops that are constitutively formed, prevents their formation by a dual action in restricting noncoding transcription and solving conflicts with replication. In agreement with this hypothesis, we have previously reported biochemical and single-molecule evidence that purified Sen1 is poorly processive in unwinding DNA:RNA duplexes (Porrua and Libri, 2013; Wang et al., 2019).

\section{Sen1 and RNases $H$ cooperate to release RNAPII at the ribosomal DNA}

At the rRFB and upstream of $R D N 5$, both the sen1-3 and the $r n h 1 \Delta r n h 201 \Delta$ mutations lead to replication-dependent, increased RNAPII occupancy, suggesting that both factors play complementary or redundant functions in the efficient removal of transcription complexes. Transcription upstream of the rRFB was previously shown to be required for inducing rDNA copy number amplification. It was shown that bi-directional transcription generated from the E-pro region using the strong GAL10 promoter releases cohesin complexes and induces rDNA amplification in a strain containing only two rDNA repeats (Kobayashi and Ganley, 2005). Because of its stability and possibly its size, it is unlikely that the transcript we describe here corresponds to the E-prom transcripts reported by Kobayashi et al. (Kobayashi and Ganley, 2005). However, it is possible that Sen 1 and RNases $\mathrm{H}$ provide an additional layer of control preventing RNAPII transcription from invading the rDNA, which could cause genomic instability in the region. It is also possible that efficiently removing RNAPII from upstream of the stalled fork is important to limit replication stress and favor the progression of replication in this region where two replication forks converge.

A direct role of RNases $\mathrm{H}$ in termination could complement the function of Sen1 when recruited by the replisome, and possibly underlie the synthetic lethality observed. In a mechanistic perspective it can be envisioned that the removal of R-loops by RNases $\mathrm{H}$ weakens the stability of the elongation complex, facilitating its dismantling at sites of conflicts. For instance, this could occur if, upon pausing, RNAPII would backtrack on a substrate on 
which RNase $\mathrm{H}$ has previously removed the DNA-associated nascent transcript engaged in an R-loop. Alternatively, it is possible that RNases $\mathrm{H}$ might favour RNAPII release at the rRFB (and possibly other sites) by cleaving the R-loop-engaged RNA thus providing an entry site for the Rat1 exonuclease, which would degrade the 3' portion of the nascent RNA and terminate transcription by the "torpedo" mechanism (Porrua et al., 2016). If Rat1 is a downstream effector of RNases $\mathrm{H}$ in at least some genomic locations, it could be expected that its mutation strongly affects growth of sen1-3 cells, phenocopying the double rnh1 $1 \Delta$ rnh2014 deletion. Indeed, associating the thermosensitive rat1-1 allele to the sen1-3 mutation induced a strong synthetic growth defect at the semi-permissive temperature for rat1-1 (Figure $\mathrm{S} 4 \mathrm{H}$, compare growth of rat1-1 to rat1-1 sen1-3 cells). This phenotype is unlikely due to defective termination of mRNA-coding genes in rat1-1 cells because the even stronger defect induced by mutation of Rna15, an essential termination factor, did not generate a similar synthetic phenotype when associated to sen1-3 (Figure S4H, compare growth of rna15-2 to rna15-2 sen1-3 cells). Interestingly, a role in transcription termination for RNase $\mathrm{H}$ is in agreement with a recent report showing that cleavage of the nascent RNA by oligonucleotidedirected digestion (possibly mimicking R-loop digestion) could induce torpedo-generated transcription termination in vivo (Lai et al., 2020). Finally, it is also possible that digestion of the DNA:RNA heteroduplex by RNase $\mathrm{H}$ provides a better opportunity for Sen 1 to efficiently access the nascent RNA close to the stalled elongation complex and induce termination. Indeed, we have previously shown that in vitro Sen1 cannot access DNA:RNA or RNA:RNA double stranded regions of the nascent transcript, the presence of which actually hampers Sen1-dependent termination when close to the RNAPII (Porrua and Libri, 2013; Xie et al., 2021). Besides reducing its binding opportunities, R-loops might also hinder the translocation on the nascent transcript of Sen1, which we have shown to have in vitro poor processivity when unwinding heteroduplexes (Porrua and Libri, 2013; Wang et al., 2019). 


\section{Sen1 resolves transcription-transcription conflicts}

We demonstrate that mutation of Sen1 has NNS termination-independent effects in many genomic regions (Figure 7), but with different modalities. In sen1-3 cells, accumulation of RNAPII at the rRFB and upstream of RDN5 is dependent on ongoing replication, is associated to the formation of R-loops and is also dependent on RNases $\mathrm{H}$.

\section{Figure 7}

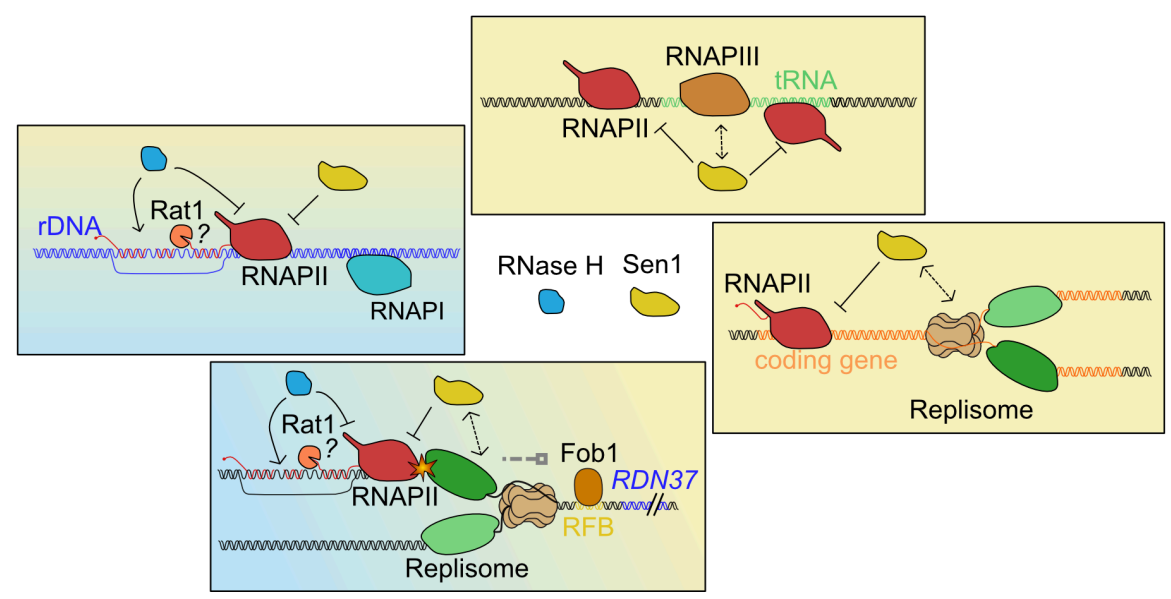

Figure 7: Model of the function of Sen1 and RNases $H$ in controlling transcription replication and transcription-transcription conflicts.

Sen1 resolves transcription-replication and transcription-transcription conflicts by dislodging RNAPII from several locations including the 5'-end of coding genes, the rRFB, the rDNA and tRNA genes. During TRCs Sen1 is recruited via interaction with the replisome, while at tRNA genes the recruitment occurs via the interaction with RNAPIII. RNases $\mathrm{H}$ assist Sen1 in limiting RNAPII at the rRFB and in the rDNA, via a mechanism that could involve Rat1.

Conversely, we did not observe a role for RNases $\mathrm{H}$, nor an effect of hsRNH1 overexpression in limiting replication-dependent RNAPII accumulation at genes, suggesting that R-loops are not formed in these TSS-proximal locations. The peak of differential RNAPII accumulation in sen1-3 cells is located roughly $100 \mathrm{nt}$ after the TSS (Figure 2A). Considering the physical occupancy of the polymerase, the binding of the capping enzymes and the 
capping complex, it is possible that the limited window of free nascent RNA does not allow efficient formation of R-loops.

At tRNA genes, although R-loops were detected at some genes upstream of paused polymerases (data not shown) RNases $\mathrm{H}$ do not appear to play a significant role, maybe because alternative mechanisms are in place cooperating with Sen1 to release the polymerase as previously shown for other sites of roadblock (Candelli et al., 2018; Colin et al., 2014). In these cases, Sen1 is solving conflicts between RNAPII and RNAPIII, by virtue of its interactions with the latter that is also lost in sen1-3, and, consistently, RNAPII accumulation is observed also in the absence of replication. The fact that the same region of Sen1 mediates alternative interactions with RNAPIII and the replisome might have important functional implications, to ensure that the two functions of the helicase, although mechanistically similar, remain distinct, and that replisome components and RNAPIII are never found, inappropriately, in the same complex, connected by Sen1. Perhaps this region of Sen1 mediates contacts with other molecular machineries to exert similar functions. In this regard, we observed marked RNAPII persistence in sen1-3 cells antisense of the RDN37 transcription unit, in correspondence of the ITS1. Limiting RNAPII in this region is also dependent on RNases $\mathrm{H}$ and prominent levels of R-loops are observed by H-CRAC. However, this accumulation was also observed in the absence of replication, and why it occurs in the sen 1-3 mutant is presently unclear. One possibility is that the interaction with another factor, responsible for recruiting Sen1 at this site, is also lost in the sen1-3 mutant and that RNAPII persists at sites of headon conflicts with RNAPI. It is enticing to speculate that Sen1 is recruited to the nucleolar site of rRNA transcription by RNAPIII, while transcribing the $5 S$ rRNA or tRNAs that are also transcribed in clustered nucleolar regions (Thompson et al., 2003; Haeusler and Engelke, 2006). Loss of Sen1 from RNAPIII complex in sen1-3 cells would also bring about defective management of RNAPI-RNAPII conflicts.

In the light of the impact of the sen1-3 mutation on RNAPIII termination, we considered the possibility that the accumulation of RNAPII at the rRFB might be linked to its role at the upstream RDN5 gene. However, RNAPIl accumulation occurs in a position that is clearly 
downstream of the region of RNAPIII readthrough, as shown by RNAPIII CRAC analyses (Figure S4A).

Together, these results, obtained in the absence of possibly interfering transcriptional defects, allow attributing to Sen1 a role of "master conflicts regulator" that is similar in many aspects to the one described for Dicer in S. pombe (Castel et al., 2014). Dicer was indeed implicated in releasing RNAPII at genes (in this case in their 3'-end), antisense of tRNA genes and rRNA transcription and at sites of replication stress. Although the mechanism of action of the two factors is unlikely to be similar, converging evolution might have hijacked existing cellular mechanism to fulfill the important role of coordinating essential cellular processes.

\section{H-CRAC is a suitable method for R-loops detection genome-wide}

We describe here a novel method to detect R-loops with unprecedented sensitivity and resolution. H-CRAC meets all the essential landmark requirements we assessed for bona fide R-loop detection (Chédin et al., 2021) strongly supporting the notion that RNase H targets detected by this method represent bona fide R-loops. It is important to stress that H-CRAC is fundamentally different from ChIP as it detects the interaction of RNases $\mathrm{H}$ with the RNA and is not expected to sense recruitment of the enzyme to the DNA in the absence of a specific contact with its targets.

Our maps are similar to published DRIP-seq maps, as witnessed by the statistically significant overlap for the R-loop-forming genes detected by $\mathrm{H}-\mathrm{CRAC}$ and the only directional map available (San Martin-Alonso et al., 2021) (Figure 4B). Comparisons with other published data (Achar et al., 2020; Wahba et al., 2016), are also statistically significant (data not shown) but are less reliable, considering the non-directional nature of these studies. Nevertheless, in many cases differences are also observed between H-CRAC and DRIP-seq (Figure 4A). Some of these differences can be accounted for by the better resolution and most likely higher sensitivity (i.e. the signal to background ratio) of H-CRAC relative to DRIP-seq (Figure 4A). However, it is also possible that the targets detected by the two methods are, to some extent, inherently different. It was suggested that short R-loops might not survive the extraction and 
immunoprecipitation steps of DRIP (Chédin et al., 2021), still they could be detected by in vivo crosslinking to RNases $\mathrm{H}$. Conversely, some stable R-loops detected by DRIP might not be efficiently recognized by RNases $\mathrm{H}$. Thus, the two methods might provide overlapping and complementary outputs for a better understanding of the distribution, metabolism and functional implications of DNA:RNA hybrids. The thorough analyses of R-loop distribution in yeast, the differences between Rnh1 and Rnh201 and the relationships with other detection methods are beyond the scope of this report and will be detailed elsewhere. However, to the light of the results and controls presented here we trust that $\mathrm{H}-\mathrm{CRAC}$ will provide invaluable information to study R-loop biology and the relationships with transcription and genome maintenance. 


\section{MATERIALS AND METHODS}

\section{Yeast strains, plasmids and oligonucleotides}

The strains, plasmids and oligonucleotides used in this study are listed in Key Resource Table. Yeast strains used in this study derive from W303 or BMA64, which is a trp1 $\Delta$ derivative of W303. Strains newly modified were constructed with standard procedures (Longtine et al., 1998).

\section{Transcription-Associated Recombination (TAR) assay}

To assess the frequency of recombination, strains of interest were transformed with the pRS314-L recombination reporter at $30^{\circ} \mathrm{C}$. Recombination events were scored by assessing the number of cells containing a functional, recombined $L E U 2$ gene relative to the total number of cells plated. Six colonies of at least three independent transformants were analysed.

\section{Cell growth for CRAC and Copy Number experiments}

For each condition, $2 \mathrm{~L}$ of cells expressing an HTP-tagged version of the protein of interest expressed either from the endogenous locus (i.e., Rpb1, Rnh1, Rnh201) or from a plasmid (hsRNH1) were grown in logarithmic phase to $\mathrm{OD}_{600}=0.6$ at $30^{\circ} \mathrm{C}$ in a CSM-TRP medium. Cells ectopically expressing the mAIRN construct were grown in CSM-Trp-Ura. Cells over-expressing hsRNH1 were grown in CSM-Trp-His.

$\mathrm{G}_{1}$ cell cycle arrest was triggered at $\mathrm{OD}_{600}=0.3$ by 3 consecutive additions of 4,8 and 4 mg of $\alpha$-factor spaced by $40 \mathrm{~min}$. $40 \mathrm{~min}$ after the last addition of $\alpha$-factor, and before UVcrosslinking, $\mathrm{G}_{1}$ arrest was verified both by microscopic visualisation of cell morphology and by flow cytometry (Figure S2J).

For analyses in S-phase, cells were arrested in $\mathrm{G}_{1}$ by $\alpha$-factor as described above and released into S-phase by removing $\alpha$ factor by filtration on a glass microfiber filter (pore $\varnothing=1.6$ $\mu \mathrm{m})$. Cells were washed while still on the filter and then resuspended in $2 \mathrm{~L}$ of fresh medium 
lacking $\alpha$-factor at $30^{\circ} \mathrm{C}$ for $30 \mathrm{~min}$ or $45 \mathrm{~min}$. Release was verified by visualisation of cell morphology and flow cytometry (Figure S2J). Two biological replicates were performed for each condition, showing high correlation (Fig S7B).

\section{UV-Crosslinking and cDNA analysis (CRAC)}

The CRAC protocol used in this study is derived from Granneman et al. (2009) with some modifications described in (Challal et al., 2018; Colin et al., 2014).

Briefly, cells were crosslinked by UV exposure for 50 seconds using a W5 UV crosslinking unit (UVO3 Ltd) and harvested by centrifugation at $4^{\circ} \mathrm{C}$. Cell pellets were washed once with ice-cold 1x PBS, weighted and resuspended in $2.4 \mathrm{~mL} /(\mathrm{g}$ of cells) of TN150 buffer (50 mM Tris $\mathrm{pH}$ 7.8, $150 \mathrm{mM} \mathrm{NaCl}, 0.1 \% \mathrm{NP}-40$ and $5 \mathrm{mM} \beta$-mercaptoethanol) supplemented with fresh protease inhibitors (AEBSF, Complete ${ }^{\text {TM }}$ EDTA-free Protease Inhibitor Cocktail, Roche). Emulsions were snap-frozen in droplets in liquid nitrogen and cells subjected to cryogenic grinding using a Ball Mill MM 400 (5 cycles of 3 minutes at $20 \mathrm{~Hz}$ ). The resulting frozen lysates were thawed on ice, treated with DNase I (165 units per gram of cells) incubated at $25^{\circ} \mathrm{C}$ for $1 \mathrm{~h}$ to solubilize the chromatin and then clarified by centrifugation at $16 \mathrm{krpm}$ for 30 $\min$ at $4^{\circ} \mathrm{C}$.

RNA-protein complexes were affinity-purified with M-280 tosylactivated dynabeads coupled with rabbit IgGs (10 mg of beads per sample), washed with TN1000 buffer (50 mM Tris $\mathrm{pH} 7.8,1 \mathrm{M} \mathrm{NaCl}, 0.1 \% \mathrm{NP}-40$ and $5 \mathrm{mM} \beta$-mercaptoethanol), and eluted by TEV protease digestion. RNAs were subjected to partial degradation by treating with $0.2 \mathrm{U}$ of Rnase cocktail (Rnace-IT, Agilent) and the reaction was stopped by the addition of guanidine$\mathrm{HCl}$ to a final concentration of $6 \mathrm{M}$. Eluates underwent then a second immobilisation on $\mathrm{Ni}$ NTA columns (Qiagen, $100 \mu \mathrm{l}$ of slurry per sample) overnight at $4^{\circ} \mathrm{C}$ and were extensively washed. Sequencing adaptors were ligated to the RNA molecules as described in the original procedure. RNA-protein complexes were eluted with elution buffer containing $50 \mathrm{mM}$ Tris $\mathrm{pH}$ 7.8, $50 \mathrm{mM} \mathrm{NaCl}, 150 \mathrm{mM}$ imidazole, $0.1 \% \mathrm{NP}-40$ and $5 \mathrm{mM} \beta$-mercaptoethanol fractionated 
using a Gel Elution Liquid Fraction Entrapment Electrophoresis (GelFree) system (Expedeon) following manufacturer's specifications. The fractions containing the protein of interest were treated with $100 \mu \mathrm{g}$ of proteinase $\mathrm{K}$, and RNAs were purified and reverse-transcribed using reverse transcriptase Superscript IV (Invitrogen).

After quantification of the recovered material via quantitative PCR, the cDNAs were amplified with an appropriate number of PCR cycles using LA Taq polymerase (Takara), and then the reactions were treated with $200 \mathrm{U} / \mathrm{mL}$ of Exonuclease I (NEB) for $1 \mathrm{~h}$ at $37^{\circ} \mathrm{C}$. Finally, the DNA was purified using NucleoSpin columns (Macherey-Nagel) and sequenced on a NextSeq 500 Illumina sequencer.

The H-CRAC protocol contained a few modifications to improve the recovery of tagged RNases H. DNase I treatment was replaced by a step of chromatin shredding by sonication in an ice-cold bath (15 min, High, $45 \mathrm{sec}$ ON/OFF, Diagenode). The GelFree fractionation was omitted to avoid loss of material because the eluate after the second purification step was judged sufficiently pure.

\section{Copy Number analysis}

An aliquot roughly corresponding to $0.2 \mathrm{~g}$ of lysate powder from the CRAC experiment was transferred to a separate tube and used to perform genomic extraction using the Genomic-tip 20/G kit (QIAGEN) following manufacturer's specifications. DNA was fragmented using sonication ( 200 to 500 bp size range). Sequencing libraries were prepared using a ThruPLEX DNA-seq kit (Rubicon Genomics). Next-generation sequencing was performed on a HiSeq 4000 (Illumina). Single-end reads of $50 \mathrm{bp}$ were aligned to the S. cerevisiae genome (2011).

\section{DNA:RNA immunoprecipitation (DRIP)}

DNA:RNA hybrid immunoprecipitation (DRIP) was performed using the S9.6 DNA:RNA hybrid-specific monoclonal antibody according to a published procedure (Mischo et al., 2011; Wahba et al., 2016), with the modifications described in (Bonnet et al., 2017). Briefly, genomic 
DNA was phenol-extracted from cells growing exponentially and isolated by ethanol precipitation. $50 \mu \mathrm{g}$ of purified nucleic acids were digested by a cocktail of restriction enzymes (EcoRI, HindIII, Xbal, Sspl, BsrGI; FastDigest enzymes; Thermo Scientific) for $30 \mathrm{~min}$ at $37^{\circ} \mathrm{C}$ in a total volume of $100 \mu \mathrm{L}$. An RNase $\mathrm{H}$ treatment (10 units, Sigma) was included in the restriction reaction of control samples to assess the specificity of the DRIP signal. Digested samples were further diluted 4-fold with FA1 buffer (0.1\% SDS, 1\% Triton, $10 \mathrm{mM} \mathrm{HEPES} \mathrm{pH}$ $7.5,0.1 \%$ sodium deoxycholate, $275 \mathrm{mM} \mathrm{NaCl}$ ) and incubated overnight at $4^{\circ} \mathrm{C}$ in the presence of $1.5 \mu \mathrm{g}$ of S9.6 purified antibody (Kerafast). Antibody-associated DNA:RNA hybrids were then captured on protein G Sepharose beads (GE Healthcare), washed and purified according to standard ChIP procedures. Input and immunoprecipitated DNA amounts were quantified by real-time PCR with a LightCycler 480 system (Roche) using SYBR Green incorporation according to the manufacturer's instructions. Oligos DL4519 and DL4520 were used to amplify the mAIRN locus while oligos DL4597 and DL4598 were used to amplify an intergenic region located in proximity to the $\mathrm{HO}$ gene and used a negative control. The amount of DNA in the immunoprecipitated fraction was divided by the amount detected in the input to evaluate the percentage of immunoprecipitation (\% of IP).

\section{Imaging of Rad52-YFP foci}

Rad52-YFP foci formation was assessed in exponentially growing cells $\left(0.5 \leq \mathrm{OD}_{600} \leq 1\right)$ in $\mathrm{CSM}$ medium at $30^{\circ} \mathrm{C}$ or $37^{\circ} \mathrm{C}$ as indicated. For wild-type Sen1-depleted conditions, Indole3'-Acetic Acid (IAA, Sigma) was supplemented at a final concentration of $500 \mu \mathrm{M} 1 \mathrm{~h}$ before imaging. Wide-field fluorescence images were acquired using a Leica DM6000B microscope with a 100X/1.4 NA (HCX Plan-Apo) oil immersion objective and a CCD camera (CoolSNAP $\mathrm{HQ}$; Photometrics). The acquisition system was piloted by the MetaMorph software (Molecular Devices). For all images, $z$ stacks sections of $0.2 \mu \mathrm{m}$ were acquired using a piezo-electric motor (LVDT; Physik Instrument) mounted underneath the objective lens. Images were scaled equivalently and z-projected using ImageJ. An average of three experiments, each of them visualizing at least 300 cells per condition, is shown in the figures. 
For thermosensitive alleles, and their relative controls, after reaching exponential growth cells were shifted to $37^{\circ} \mathrm{C}$ by the addition of pre-warmed media, and incubated for $1 \mathrm{~h}$ before imaging.

\section{Protein analyses}

Proteins levels were analysed using current methodologies.

\section{Dataset processing and data analysis}

\section{CRAC}

CRAC datasets were analysed as described (Candelli et al., 2018; Challal et al., 2018). The pyCRAC script pyFastqDuplicateRemover was used to collapse PCR duplicates using a 6 nucleotides random tag included in the 3' adaptor (see Key Resources Table). The resulting sequences were reverse complemented with Fastx reverse complement (part of the fastx toolkit, http://hannonlab.cshl.edu/ fastx_toolkit/) and mapped to the R64 genome (Cherry et al., 2012) with bowtie2 (-N 1) (Langmead and Salzberg, 2012).

\section{Quantification and statistical analysis}

The vast majority of the analyses were performed with inhouse scripts in the $R$ studio environment. Sen1-AID CRAC datasets were analysed using the Galaxy web platform at usegalaxy.org (Afgan et al., 2018).

For all RNAPII CRAC data, the working group of 3000 genes with the highest expression level was selected by computing HT-seq count normalised to the size of the gene. This allowed excluding from our analysis genes with very low or background signal, which are potential source of computational biases.

The skew index was defined as the ratio between the RNAPII CRAC signals in the windows $[0 ;+200] /[+300 ;+500]$ relative to the TSS for each gene. A sen 1-3/WT skew index ratio was used to select genes with increased 5'-end RNAPII accumulation in the mutant 
relative to the wild-type. All features with a skew index ratio exceeding the mean plus one standard deviation of the distribution were considered to be affected.

For Copy Number analysis, all regions with a score $>1$ were considered as undergoing replication. For the selection of the "already replicated genes" genes overlapping with a replicated region with a copy number score exceeding the $95^{\text {th }}$ percentile were chosen.

When average values were represented, error bars indicate standard deviation. T tests were used to compare distributions and p-values are indicated.

\section{KEY RESOURCE TABLE}

\begin{tabular}{|c|c|c|}
\hline Antibodies & & \\
\hline Reagent or Resource & Source & Identifier \\
\hline IgG from rabbit serum & Sigma-Aldrich & Cat\# I5006; RRID: AB_1163659 \\
\hline Mouse anti Flag & Sigma-Aldrich & Cat\# F1804; RRID: AB_262044 \\
\hline $\begin{array}{l}\text { Rabbit Peroxidase Anti- } \\
\text { Peroxidase }\end{array}$ & Sigma-Aldrich & Cat\# P1291; RRID: AB_1079562 \\
\hline $\begin{array}{l}\text { Anti-phospho Histone H2A } \\
\text { (Ser129) }\end{array}$ & Merck & Cat\# 07-745-I \\
\hline Anti-DNA:RNA hybrids (S9.6) & Kerafast & $\begin{array}{l}\text { Cat\# ENH001; RRID: } \\
\text { AB_2687463 }\end{array}$ \\
\hline Goat anti-rabbit IgG-HRP & Santa Cruz & Cat\# sc-2004; RRID: AB_631746 \\
\hline
\end{tabular}

\section{Chemicals, Peptides, and Recombinant Proteins}

\begin{tabular}{|l|l|l|}
\hline Reagent or Resource & Source & Identifier \\
\hline $\begin{array}{l}\text { cOmplete EDTA-free protease } \\
\text { inhibitor cocktail tablets }\end{array}$ & Sigma-Aldrich (Roche) & Cat\# 11873580001 \\
\hline Pefabloc SC-Protease-Inhibitor & Carl Roth & \\
\hline Dnase I recombinant, Rnase-free & Sigma-Aldrich (Roche) & Cat\# A154.3 \\
\hline
\end{tabular}




\begin{tabular}{|c|c|c|}
\hline Dynabeads M-280 Tosylactivated & Thermo Fisher Scientific & Cat\# 14204 \\
\hline Recombinant GST-TEV protease & (Challal et al., 2018) & $\mathrm{N} / \mathrm{A}$ \\
\hline Rnace-It Ribonuclease Cocktail & Agilent & Cat\# 400720 \\
\hline Guanidine hydrochloride & Sigma-Aldrich & Cat\# G4505 \\
\hline Ni-NTA Agarose & QIAGEN & Cat\# 30230 \\
\hline Imidazole & Sigma-Aldrich & Cat\# 10125 \\
\hline $\begin{array}{l}\text { RNaseOUT Recombinant } \\
\text { Ribonuclease Inhibitor }\end{array}$ & Thermo Fisher Scientific & Cat\# 10777019 \\
\hline T4 RNA Ligase 2, truncated KQ & NEB & Cat\# M0373L \\
\hline T4 Polynucleotide Kinase & NEB & Cat\# M0201L \\
\hline T4 RNA Ligase 1 (ssRNA Ligase) & NEB & Cat\# M0204L \\
\hline $\begin{array}{l}\text { Proteinase } \mathrm{K} \text {, recombinant, PCR } \\
\text { grade }\end{array}$ & Sigma-Aldrich (Roche) & Cat\# 03115887001 \\
\hline $\begin{array}{l}\text { SuperScript IV Reverse } \\
\text { Transcriptase }\end{array}$ & Thermo Fisher Scientific & Cat\# 18090050 \\
\hline Rnase H & NEB & Cat\# M0297S \\
\hline Exonuclease I & NEB & Cat\# M0293S \\
\hline LA Taq & Takara & Cat\# RR002M \\
\hline FastDigest EcoRI & Thermo Fisher Scientific & Cat\# FD0275 \\
\hline FastDigest HindIII & Thermo Fisher Scientific & Cat\# FD0505 \\
\hline FastDigest Xbal & Thermo Fisher Scientific & Cat\# FD0685 \\
\hline FastDigest Sspl & Thermo Fisher Scientific & Cat\# FD0774 \\
\hline FastDigest Bsp1407I (BsrGI) & Thermo Fisher Scientific & Cat\# FD0934 \\
\hline Rnase H & Sigma-Aldrich (Roche) & Cat\# 10786357001 \\
\hline Protein G Sepharose Fast Flow & GE Healthcare & Cat\# 17061801 \\
\hline Hydroxyurea $(\mathrm{HU})$ & Sigma-Aldrich & Cat\# H8627 \\
\hline Methyl methanesulfonate (MMS) & Sigma-Aldrich & Cat\# 129925 \\
\hline$\alpha$-factor & BIOTEM & $\mathrm{N} / \mathrm{A}$ \\
\hline Paraformaldehyde & VWR Chemicals & Cat\# 28794.295 \\
\hline 3-Indoleacetic acid (IAA) & Sigma-Aldrich & Cat\# I2886 \\
\hline
\end{tabular}




\begin{tabular}{|c|c|c|c|}
\hline \multicolumn{4}{|l|}{ Strains } \\
\hline Lab number & Identifier & Source & Genotype \\
\hline DLY671 & Wild-type & F. Lacroute & as BMA64; Mat a \\
\hline DLY128 & rna15-2 & F. Lacroute & as W303; rna15-2; Mat a \\
\hline DLY753 & rat1-1 & F. Lacroute & as BMA64; rat1-1; Mat alpha \\
\hline DLY2057 & sen1-1 & F. Lacroute & as BMA64; sen1-1; Mat a \\
\hline DLY2571 & Rpb1-HTP & $\begin{array}{l}\text { (Candelli et al., } \\
\text { 2018) }\end{array}$ & as BMA64; RPB1::HTP::TRP1kl; Mat a \\
\hline DLY3173 & sen1-3-TAP & $\begin{array}{l}\text { (Appanah et al., } \\
\text { 2020) }\end{array}$ & $\begin{array}{l}\text { as W303; sen1 W773A E774A } \\
\text { W777A::TAP::KanMX; Mat a }\end{array}$ \\
\hline DLY3197 & sen1-3 & This study & as W303; sen1 W773A E774A W777A; Mat a \\
\hline DLY3211 & Rpb1-HTP sen1-3 & This study & $\begin{array}{l}\text { as BMA64; sen1 W773A E774A W777A; } \\
\text { RPB1:HTP::TRP1kl; Mat a }\end{array}$ \\
\hline DLY3321 & Rnh1-HTP & This study & as BMA64; RNH1::HTP::TRP1kl; Mat a \\
\hline DLY3348 & Rnh1-HTP sen1-3 & This study & $\begin{array}{l}\text { as BMA64; RNH1::HTP::TRP1kl; sen1 W773A } \\
\text { E774A W777A; Mat a }\end{array}$ \\
\hline DLY3368 & sen1-3-TAP rat1-1 & This study & $\begin{array}{l}\text { as BMA64; sen1 W773A E774A } \\
\text { W777A::TAP::KanMX; rat1-1; Mat a }\end{array}$ \\
\hline DLY3370 & sen1-3-TAP rat15-2 & This study & $\begin{array}{l}\text { as BMA64; sen1 W773A E774A } \\
\text { W777A::TAP::KanMX; rna15-2; Mat a }\end{array}$ \\
\hline DLY3421 & Rpb1-HTP rnh14 rnh2014 & This study & $\begin{array}{l}\text { as BMA64; rnh1::hphNT; rnh20http:isMX; } \\
\text { RPB1::HTP::TRP1kl; Mat a }\end{array}$ \\
\hline DLY3432 & Rnh201-HTP & This study & as BMA64; RNH201::HTP::TRP1kl; Mat a \\
\hline DLY3438 & Rnh201-HTP sen1-3 & This study & $\begin{array}{l}\text { as BMA64; RNH201::HTP::TRP1kl; sen1 } \\
\text { W773A E774A W777A; Mat a }\end{array}$ \\
\hline DLY3443 & Rad52-YFP & (Lisby et al., 2001) & as BMA64; RAD52::YFP; bar1::LEU2; Mat a \\
\hline DLY3477 & $\begin{array}{l}\text { Rad52-YFP Sen1-AID } \\
\text { pFL38-sen1-3 }\end{array}$ & This study & $\begin{array}{l}\text { as BMA64; SEN1::AID::KAN::OsTIR1; } \\
\text { RAD52::YFP; pFL38-sen1-3::URA; Mat a }\end{array}$ \\
\hline DLY3479 & 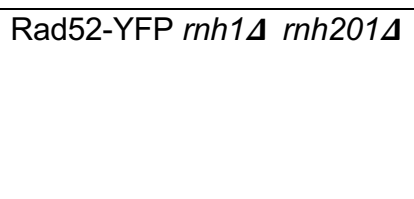 & This study & $\begin{array}{l}\text { as BMA64; rnh1::hphNT; rnh20http:isMX; } \\
\text { RAD52::YFP; RPB1::HTP::TRPkl; bar1::LEU2; } \\
\text { Mat a }\end{array}$ \\
\hline
\end{tabular}




\begin{tabular}{|l|l|l|l|}
\hline DLY3481 & Rad52-YFP Sen1-AID rnh14 & This study & $\begin{array}{l}\text { as BMA64; SEN1::AID::KAN::OsTIR1; } \\
\text { rnh1::hphNT; rhttp:1::HisMX; RAD52::YFP; } \\
\end{array}$ \\
& Rh2014 + pFL38-sen1-3 & & $\begin{array}{l}\text { RPB1::HTP::TRPkl; bar1::LEU2; pFL38-sen1- } \\
3:: U R A ; \text { Mat a }\end{array}$ \\
\hline DLY3562 & Rad52-YFP sen1-3 & This study & As W303, sen1-3, RAD52::YFP \\
\hline DLY3582 & Rad52-YFP sen1-1 & This study & As W303, sen1-1, RAD52::YFP \\
\hline DLY3583 & Rad52-YFP nrd1-102 & This study & As W303, nrd1-102, RAD52::YFP \\
\hline DLY3584 & Rad52-YFP sen1-3 nrd1-102 & This study & As W303, nrd1-102, sen1-3, RAD52::YFP \\
\hline
\end{tabular}

\begin{tabular}{|c|c|c|}
\hline \multicolumn{3}{|l|}{ Critical commercial assay } \\
\hline Reagent or Resource & Source & Identifier \\
\hline Genomic-tip 20/G kit & QUIAGEN & Cat\# 10223 \\
\hline $\begin{array}{l}\text { LightCycler } 480 \text { SYBR Green I } \\
\text { Master }\end{array}$ & Roche & Cat\# 04887352001 \\
\hline $\begin{array}{l}\text { NucleoSpin Gel and PCR Clean- } \\
\text { up }\end{array}$ & Macherey-Nagel & Cat\# 740609 \\
\hline Pierce Spin Columns - Snap Cap & Thermo Fisher Scientific & Cat\# 69725 \\
\hline Vivacon 500 & Sartorius & Cat\# VN01H22 \\
\hline Qubit dsDNA HS Assay Kit & $\begin{array}{l}\text { Thermo Fisher Scientific } \\
\text { (Invitrogen) }\end{array}$ & Cat\# Q32851 \\
\hline $\begin{array}{l}\text { SuperSignal West Pico } \\
\text { Chemiluminescent Substrate }\end{array}$ & Thermo Fisher Scientific & Cat\# 34080 \\
\hline
\end{tabular}

\begin{tabular}{|c|c|c|}
\hline \multicolumn{3}{|l|}{ Plasmids } \\
\hline Reagent or Resource & Source & Identifier \\
\hline pRS314-L & (Prado and Aguilera, 2005) & $\mathrm{N} / \mathrm{A}$ \\
\hline $\begin{array}{l}\text { pDL983-pCM190-mAIRN- } \\
\text { 350to846-pGAL-CUP1 }\end{array}$ & This study & $\mathrm{N} / \mathrm{A}$ \\
\hline
\end{tabular}




\begin{tabular}{|l|l|l|}
\hline pDL987-pRS424-GPDprom-hs- & (Wahba et al, 2011) & N/A \\
H1 & & N/A \\
\hline $\begin{array}{l}\text { pDL1010-pRS424-GPDprom-PTH- } \\
\text { hsRNH1 }\end{array}$ & This study & \\
\hline
\end{tabular}

\begin{tabular}{|l|l|l|}
\hline \multicolumn{2}{|l|}{ Software and Algorithms } & Identifier \\
\hline Reagent or Resource & Source & RSID:SCR_000432 \\
\hline RStudio & NIH & $\begin{array}{l}\text { https://imagej.nih.gov/ii]; } \\
\text { RRID:SCR_003070 }\end{array}$ \\
\hline ImageJ & Serif & $\begin{array}{l}\text { https://affinity.serif.com/en- } \\
\text { us/designer/; RRID:SCR_016952 }\end{array}$ \\
\hline Affinity Designer & & \\
\hline
\end{tabular}

\begin{tabular}{|l|l|l|}
\hline \multicolumn{2}{|l|}{ Oligonucleotides } & Source \\
\hline Reagent or Resource & This study & N/A \\
\hline GL4519- & & N/A \\
\hline $\begin{array}{l}\text { DL4520- } \\
\text { CAACTCTCCAGCAGCGTGGT }\end{array}$ & This study & N/A \\
\hline DL4597- & This study & N/A \\
\hline $\begin{array}{l}\text { DL4598- } \\
\text { AGCTTCTGCAAACCTCATTTG }\end{array}$ & This study & \\
\hline
\end{tabular}

\section{DATA AVAILABILITY}

All the datasets generate by this study have been deposited in the Geo Expression Omnibus repository at NCBI and are available using the code GSE195936. 


\section{ACKNOWLEDGEMENTS}

We wish to thank Andres Aguilera, Doug Koshland, Rodney Rothstein for providing strains or plasmids; Giacomo De Piccoli, Sarah Lambert, Vincent Vanoosthuyse, Michel Werner, Frédéric Chédin, Julien Gros and the members of the Libri and Palancade labs for critical reading of the manuscript and fruitful discussion; Vasudha Sharma for help with the generation of mutants and with microscopy experiments. We also wish to thank Yan Jaszczyszyn for expert technical help in preparing CRAC libraries for sequencing. This work has benefited from the facilities and expertise of the high throughput sequencing core facility of I2BC (Centre de Recherche de Gif - http://www.i2bc-saclay.fr/).

\section{AUTHOR CONTRIBUTION}

UA and DL designed experiments. UA performed all the experiments and analysis excluding the raw data analysis of the DNA Copy number and the Sen1-AID RNAPII CRAC and the ensuing bioinformatic analysis presented in Figure 1. DC performed the Sen1-AID RNAPII CRAC. GW offered technical assistance for several experiments. AL assisted UA with the DNA Copy Number analysis. BP assisted UA with DRIP experiments and Rad52 foci imaging. RA provided the sen1-3 strain ahead of publication. DL performed the bioinformatic analysis presented in Figure 1. UA and DL wrote the manuscript. DL supervised the project. DL collected funding. All authors provided feedbacks during the writing and approved the manuscript.

\section{DECLARATION OF INTERESTS}

The authors declare no competing interests. 


\section{FUNDING}

This work was supported by the Centre National de la Recherche Scientifique (C.N.R.S.), the Fondation pour la Recherche Medicale (F.R.M., programme Equipes 2019 to D.L.), l'Agence National pour la Recherche (ANR-16-CE12-0022-01 to D.L. and P.P.; ANR21-CE12-0040-01 to D.L. and B.P.; ANR-18-CE12-0003 to B.P.; ANR-19-CE12-0016-01 to P.P.), the Fondation ARC pour la Recherche sur le Cancer and the Ligue Nationale contre le Cancer to B.P. and the IdEx Université de Paris (ANR-18-IDEX-0001 to B.P.).

U.A. was supported by the French Ministry for Education and Research, by the Fondation ARC pour la Recherche sur le Cancer and the EUR G.E.N.E. (reference \#ANR-17EURE-0013), which is part of the Universite de Paris IdEx \#ANR-18-IDEX-0001 funded by the French Government through its "Investments for the Future" program. 


\section{REFERENCES}

Achar, Y.J., Adhil, M., Choudhary, R., Gilbert, N., and Foiani, M. (2020). Negative supercoil at gene boundaries modulates gene topology. Nature 577, 701-705.

Afgan, E., Baker, D., Batut, B., van den Beek, M., Bouvier, D., Cech, M., Chilton, J., Clements, D., Coraor, N., Grüning, B.A., et al. (2018). The Galaxy platform for accessible, reproducible and collaborative biomedical analyses: 2018 update. Nucleic Acids Res. 46, W537-W544.

Alzu, A., Bermejo, R., Begnis, M., Lucca, C., Piccini, D., Carotenuto, W., Saponaro, M., Brambati, A., Cocito, A., Foiani, M., et al. (2012). Senataxin associates with replication forks to protect fork integrity across RNA-polymerase-II-transcribed genes. Cell 151, 835-846.

Appanah, R., Lones, E.C., Aiello, U., Libri, D., and De Piccoli, G. (2020). Sen1 Is Recruited to Replication Forks via Ctf4 and Mrc1 and Promotes Genome Stability. Cell Rep. 30, 20942105.e9.

Bohnsack, M.T., Tollervey, D., and Granneman, S. (2012). Identification of RNA helicase target sites by UV cross-linking and analysis of cDNA. Methods Enzymol. 511, 275-288.

Bonnet, A., Grosso, A.R., Elkaoutari, A., Coleno, E., Presle, A., Sridhara, S.C., Janbon, G., Géli, V., de Almeida, S.F., and Palancade, B. (2017). Introns Protect Eukaryotic Genomes from Transcription-Associated Genetic Instability. Mol. Cell 67, 608-621.e6.

Candelli, T., Challal, D., Briand, J.-B., Boulay, J., Porrua, O., Colin, J., and Libri, D. (2018). Highresolution transcription maps reveal the widespread impact of roadblock termination in yeast. EMBO J. 37.

Carrasco-Salas, Y., Malapert, A., Sulthana, S., Molcrette, B., Chazot-Franguiadakis, L., Bernard, P., Chédin, F., Faivre-Moskalenko, C., and Vanoosthuyse, V. (2019). The extruded non-template strand determines the architecture of R-loops. Nucleic Acids Res. 47, 67836795.

Castel, S.E., Ren, J., Bhattacharjee, S., Chang, A.-Y., Sánchez, M., Valbuena, A., Antequera, F., and Martienssen, R.A. (2014). Dicer promotes transcription termination at sites of replication stress to maintain genome stability. Cell 159, 572-583.

Challal, D., Barucco, M., Kubik, S., Feuerbach, F., Candelli, T., Geoffroy, H., Benaksas, C., Shore, D., and Libri, D. (2018). General Regulatory Factors Control the Fidelity of Transcription by Restricting Non-coding and Ectopic Initiation. Mol. Cell 72, 955-969.e7.

Chédin, F., Hartono, S.R., Sanz, L.A., and Vanoosthuyse, V. (2021). Best practices for the visualization, mapping, and manipulation of R-loops. EMBO J. 40, e106394.

Cherry, J.M., Hong, E.L., Amundsen, C., Balakrishnan, R., Binkley, G., Chan, E.T., Christie, K.R., Costanzo, M.C., Dwight, S.S., Engel, S.R., et al. (2012). Saccharomyces Genome Database: the genomics resource of budding yeast. Nucleic Acids Res. 40, D700-705.

Churchman, L.S., and Weissman, J.S. (2011). Nascent transcript sequencing visualizes transcription at nucleotide resolution. Nature 469, 368-373. 
Colin, J., Candelli, T., Porrua, O., Boulay, J., Zhu, C., Lacroute, F., Steinmetz, L.M., and Libri, D. (2014). Roadblock termination by reb1p restricts cryptic and readthrough transcription. Mol. Cell 56, 667-680.

Costantino, L., and Koshland, D. (2018). Genome-wide Map of R-Loop-Induced Damage Reveals How a Subset of R-Loops Contributes to Genomic Instability. Mol. Cell 71, 487497.e3.

Delan-Forino, C., Schneider, C., and Tollervey, D. (2017). Transcriptome-wide analysis of alternative routes for RNA substrates into the exosome complex. PLoS Genet. 13, e1006699.

Feng, Y., Seija, N., Di Noia, J.M., and Martin, A. (2020). AID in Antibody Diversification: There and Back Again. Trends Immunol. 41, 586-600.

Ginno, P.A., Lott, P.L., Christensen, H.C., Korf, I., and Chédin, F. (2012). R-loop formation is a distinctive characteristic of unmethylated human CpG island promoters. Mol. Cell 45, 814825.

Granneman, S., Kudla, G., Petfalski, E., and Tollervey, D. (2009). Identification of protein binding sites on U3 snoRNA and pre-rRNA by UV cross-linking and high-throughput analysis of cDNAs. Proc. Natl. Acad. Sci. 106, 9613-9618.

Haeusler, R.A., and Engelke, D.R. (2006). Spatial organization of transcription by RNA polymerase III. Nucleic Acids Res. 34, 4826-4836.

Han, Z., Libri, D., and Porrua, O. (2017). Biochemical characterization of the helicase Sen1 provides new insights into the mechanisms of non-coding transcription termination. Nucleic Acids Res. 45, 1355-1370.

Haruki, H., Nishikawa, J., and Laemmli, U.K. (2008). The anchor-away technique: rapid, conditional establishment of yeast mutant phenotypes. Mol. Cell 31, 925-932.

Hazelbaker, D.Z., Marquardt, S., Wlotzka, W., and Buratowski, S. (2012). Kinetic competition between RNA Polymerase II and Sen1-dependent transcription termination. Mol Cell 49, 5566.

Kanagaraj, R., Mitter, R., Kantidakis, T., Edwards, M.M., Benitez, A., Chakravarty, P., Fu, B., Becherel, O., Yang, F., Lavin, M.F., et al. (2022). Integrated genome and transcriptome analyses reveal the mechanism of genome instability in ataxia with oculomotor apraxia 2. Proc. Natl. Acad. Sci. 119.

Kawauchi, J., Mischo, H., Braglia, P., Rondon, A., and Proudfoot, N.J. (2008). Budding yeast RNA polymerases I and II employ parallel mechanisms of transcriptional termination. Genes Dev. 22, 1082-1092.

Kobayashi, T. (2003). The replication fork barrier site forms a unique structure with Fob1p and inhibits the replication fork. Mol. Cell. Biol. 23, 9178-9188. 
Kobayashi, T., and Ganley, A.R.D. (2005). Recombination regulation by transcription-induced cohesin dissociation in rDNA repeats. Science 309, 1581-1584.

Lai, F., Damle, S.S., Ling, K.K., and Rigo, F. (2020). Directed RNase H Cleavage of Nascent Transcripts Causes Transcription Termination. Mol. Cell.

Langmead, B., and Salzberg, S.L. (2012). Fast gapped-read alignment with Bowtie 2. Nat. Methods 9, 357-359.

Leonaitè, B., Han, Z., Basquin, J., Bonneau, F., Libri, D., Porrua, O., and Conti, E. (2017). Sen1 has unique structural features grafted on the architecture of the Upf1-like helicase family. EMBO J. 36, 1590-1604.

Lisby, M., Rothstein, R., and Mortensen, U.H. (2001). Rad52 forms DNA repair and recombination centers during S phase. Proc. Natl. Acad. Sci. 98, 8276-8282.

Longtine, M.I., Demarini, S., Wach, B., and Philippsen, P. (1998). Additional modules for versatile and economical PCR-based gene deletion and modification in Saccharomyces cerevisiae. 9.

Mayer, A., Lidschreiber, M., Siebert, M., Leike, K., Soding, J., and Cramer, P. (2011). Uniform transitions of the general RNA polymerase II transcription complex. Nat Struct Mol Biol 17, 1272-1278.

Mendoza-Ochoa, G.I., Barrass, J.D., Terlouw, B.R., Maudlin, I.E., de Lucas, S., Sani, E., Aslanzadeh, V., Reid, J.A.E., and Beggs, J.D. (2019). A fast and tuneable auxin-inducible degron for depletion of target proteins in budding yeast. Yeast Chichester Engl. 36, 75-81.

Mischo, H.E., Gomez-Gonzalez, B., Grzechnik, P., Rondon, A.G., Wei, W., Steinmetz, L., Aguilera, A., and Proudfoot, N.J. (2011). Yeast Sen1 helicase protects the genome from transcription-associated instability. Mol Cell 41, 21-32.

Nishimura, K., Fukagawa, T., Takisawa, H., Kakimoto, T., and Kanemaki, M. (2009). An auxinbased degron system for the rapid depletion of proteins in nonplant cells. Nat. Methods 6, 917-922.

Porrua, O., and Libri, D. (2013). A bacterial-like mechanism for transcription termination by the Sen $1 p$ helicase in budding yeast. Nat. Struct. Mol. Biol. 20, 884-891.

Porrua, O., Boudvillain, M., and Libri, D. (2016). Transcription Termination: Variations on Common Themes. Trends Genet. TIG 32, 508-522.

Prado, F., and Aguilera, A. (2005). Impairment of replication fork progression mediates RNA polll transcription-associated recombination. EMBO J. 24, 1267-1276.

Rossi, M.J., Kuntala, P.K., Lai, W.K.M., Yamada, N., Badjatia, N., Mittal, C., Kuzu, G., Bocklund, K., Farrell, N.P., Blanda, T.R., et al. (2021). A high-resolution protein architecture of the budding yeast genome. Nature 592, 309-314. 
San Martin-Alonso, M., Soler-Oliva, M.E., García-Rubio, M., García-Muse, T., and Aguilera, A. (2021). Harmful R-loops are prevented via different cell cycle-specific mechanisms. Nat.

Commun. 12, 4451.

Schaughency, P., Merran, J., and Corden, J.L. (2014). Genome-wide mapping of yeast RNA polymerase II termination. PLoS Genet. 10, e1004632.

Schulz, D., Schwalb, B., Kiesel, A., Baejen, C., Torkler, P., Gagneur, J., Soeding, J., and Cramer, P. (2013). Transcriptome surveillance by selective termination of noncoding RNA synthesis.

Cell 155, 1075-1087.

Steinmetz, E.J., Warren, C.L., Kuehner, J.N., Panbehi, B., Ansari, A.Z., and Brow, D.A. (2006). Genome-wide distribution of yeast RNA polymerase II and its control by Sen1 helicase. Mol Cell 24, 735-746.

Thompson, M., Haeusler, R.A., Good, P.D., and Engelke, D.R. (2003). Nucleolar Clustering of Dispersed tRNA Genes. Science 302, 1399-1401.

Tye, B.W., Commins, N., Ryazanova, L.V., Wühr, M., Springer, M., Pincus, D., and Churchman, L.S. (2019). Proteotoxicity from aberrant ribosome biogenesis compromises cell fitness. ELife 8, e43002.

Wahba, L., Costantino, L., Tan, F.J., Zimmer, A., and Koshland, D. (2016). S1-DRIP-seq identifies high expression and polyA tracts as major contributors to R-loop formation. Genes Dev. 30, 1327-1338.

Wang, S., Han, Z., Libri, D., Porrua, O., and Strick, T.R. (2019). Single-molecule characterization of extrinsic transcription termination by Sen1 helicase. Nat. Commun. 10, 1545.

Xie, J., Aiello, U., Clement, Y., Haidara, N., Girbig, M., Schmitzova, J., Pena, V., Müller, C.W., Libri, D., and Porrua, O. (2021). An integrated model for termination of RNA polymerase III transcription.

Zardoni, L., Nardini, E., Brambati, A., Lucca, C., Choudhary, R., Loperfido, F., Sabbioneda, S., and Liberi, G. (2021). Elongating RNA polymerase II and RNA:DNA hybrids hinder fork progression and gene expression at sites of head-on replication-transcription collisions. Nucleic Acids Res. 49, 12769-12784.

Zencir, S., Dilg, D., Rueda, M.P., Shore, D., and Albert, B. (2020). Mechanisms coordinating ribosomal protein gene transcription in response to stress. Nucleic Acids Res. 48, 1140811420. 
bioRxiv preprint doi: https://doi.org/10.1101/2022.02.09.479708; this version posted February 10, 2022. The copyright holder for this

preprint (which was not certified by peer review) is the author/funder, who has granted bioRxiv a license to display the preprint in perpetuity. It is made available under aCC-BY-NC-ND 4.0 International license.

\section{Supplemental Figures}




\section{Figure $\mathrm{S} 1$ related to Figure 1}
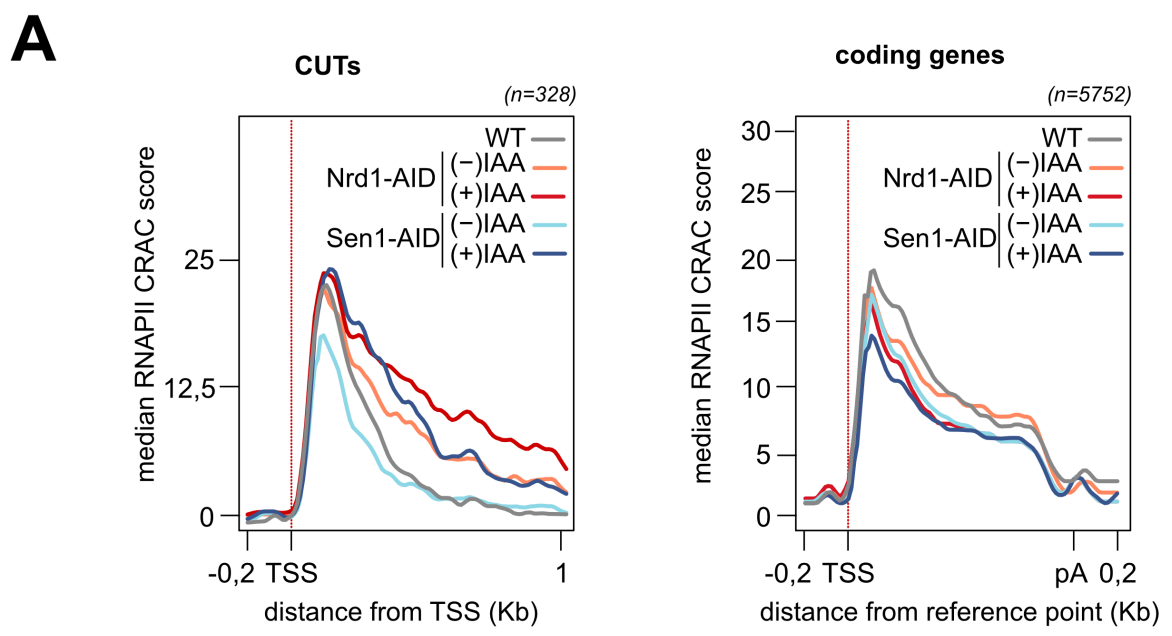

B
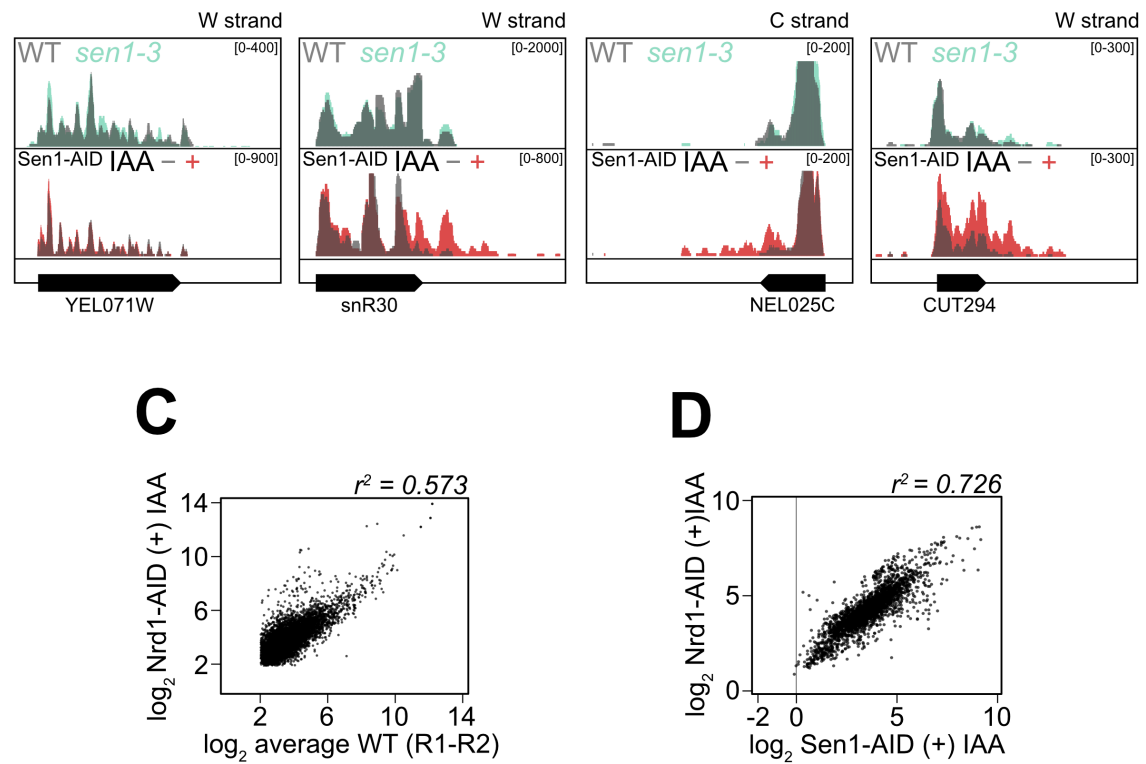

\section{Supplementary Figure 1 related to Figure 1:}

A) Metagene analysis of RNAPII distribution at a subset of validated Cryptic Unstable Transcripts (CUTs, left panel) and at coding genes (right panel) aligned at their Transcription Start Site (TSS) in wild-type (WT), Nrd1-AID and Sen1-AID cells in presence or absence of auxin (IAA). Values on the y-axis correspond to the median coverage. Note that the 3 '-end of CUTs is not well defined, hence the increase in RNAPII occupancy upon depletion of Nrd1 or Sen1 is spread over a large region. B) Representative snapshots illustrating the absence of termination defects at coding and non-coding genes in sen 1-3 cells. For comparison, the 
tracks derived from Sen1-AID cells in presence or absence of auxin (IAA), showing readthrough at CUTs and snoRNAs are also included. YEL071W is shown as a representative example of lack of termination defects at coding genes in Sen1-AID cells even in presence of IAA. C) Scatter plot as in Figure 1A but for Nrd1-AID strain under depletion conditions (auxin added for 1 hour). D) Scatter plot analysis showing the good correlation of RNAPII CRAC density in mRNA coding genes in Sen1- and Nrd1-depleted cells. 


\section{Figure S2 related to Figure 2}
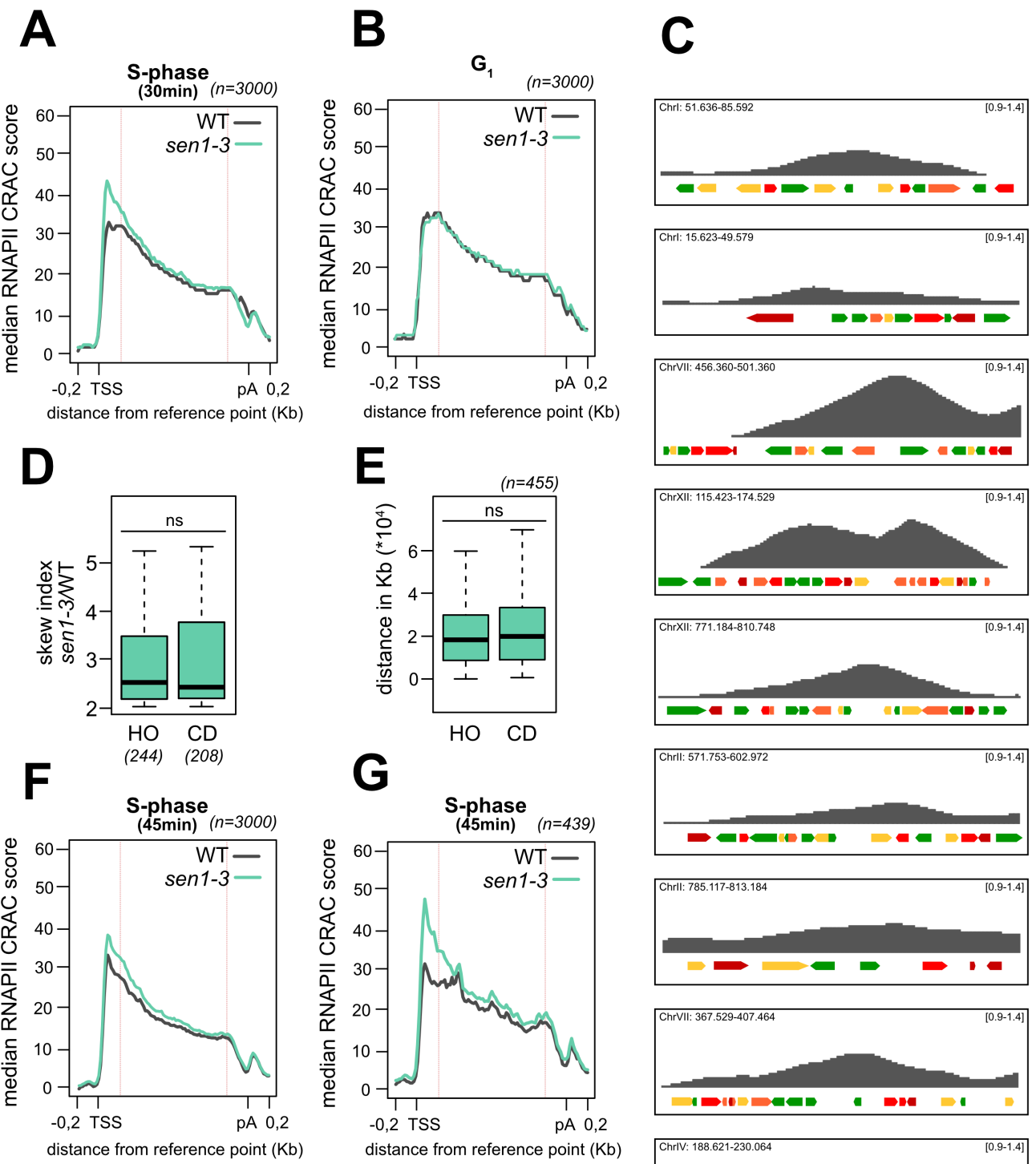

\section{H}
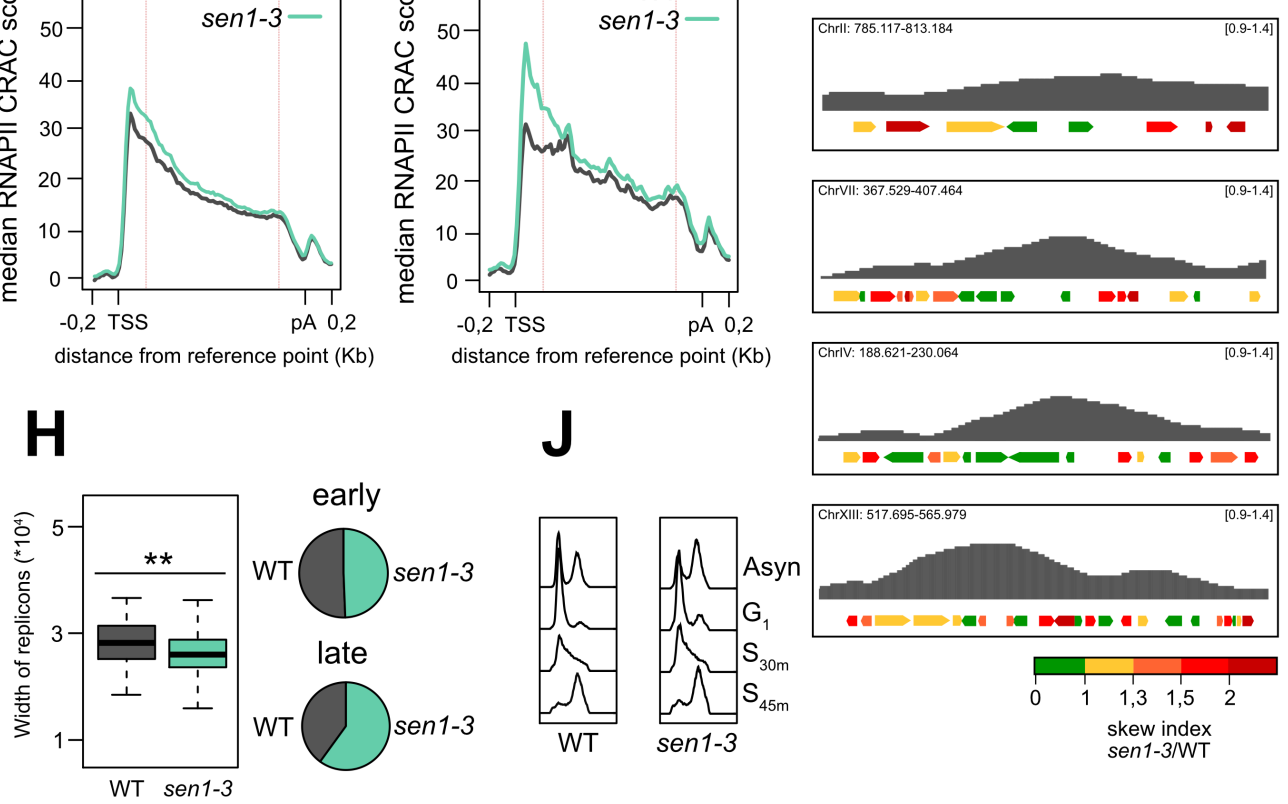


\section{Supplementary Figure 2 related to Figure 2:}

A) and B) As in Figure 2A, but on cells synchronously released in S-phase and collected 30 min after replication onset or arrested in $G_{1}$, respectively. The 3000 genes with the highest transcription levels have been used. C) As in Figure 2F, additional representative snapshots illustrating the frequent co-localisation of the replication forks (minima of the DNA copy number signal) and genes displaying a 5'-end skewed RNAPII distribution pattern in sen 1-3 cells (coloured in red). D) Boxplot comparison of the skew index ratio (sen1-3/WT) of RNAPII signal for genes oriented head-on $(\mathrm{HO})$ or in co-direction $(\mathrm{CD})$ relative to the position on the nearest activated ARS as detected by DNA copy number analysis. Genes in both groups have a replication-dependent accumulation of RNAPII in their 5'-end. E) Distribution of distances for each affected gene from the closest $\mathrm{HO}$ and $\mathrm{CD}$ origins. Affected genes are not preferentially replicated in one configuration over the other. F) Meta-analyses as in Figure 2A, but on cells synchronously released in S-phase and collected $45 \mathrm{~min}$ after replication onset. G) As in Figure 2C, but on cells synchronously released in S-phase and collected $45 \mathrm{~min}$ after replication onset. The analysis was performed on the subset of genes affected at this time point. H) Left: comparison of the width of the replicons detected by DNA copy number analysis from wild-type (WT) and sen1-3 cells synchronously grown in S-phase and collected 30 min after replication onset. ${ }^{* *} p<0.01$. Right: pies indicating the total number of activated origins that were retrieved from DNA copy number analysis in wild-type (WT) and sen1-3 cells. Origins were divided in early and late according to their replication timing in wild-type cells. J) Examples of cell cycle analysis by flow cytometry from the cells used for the experiments shown in Figure 2 and S2. 


\section{Figure S3 related to Figure 4}

\section{A}

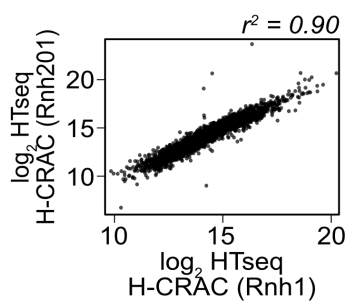

D
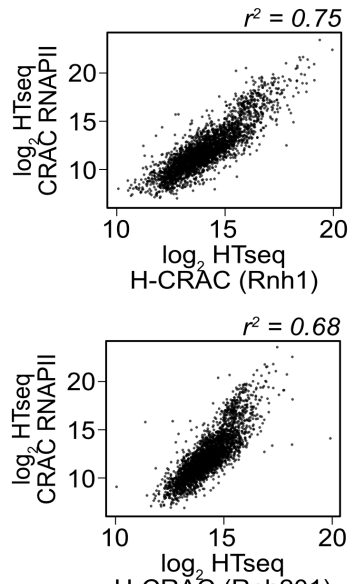

$\mathbf{F}$

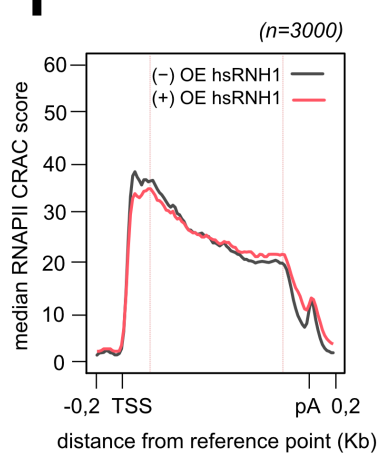

H

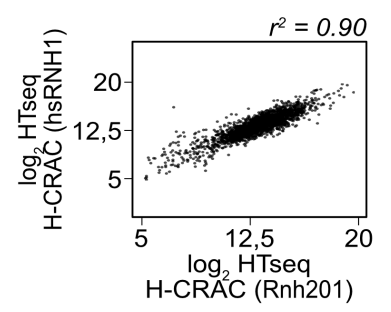

\section{B}

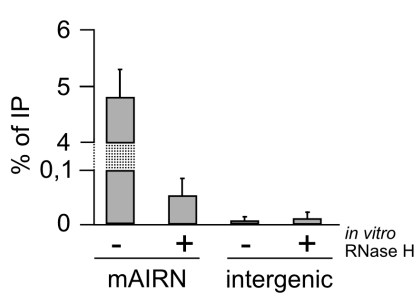

E

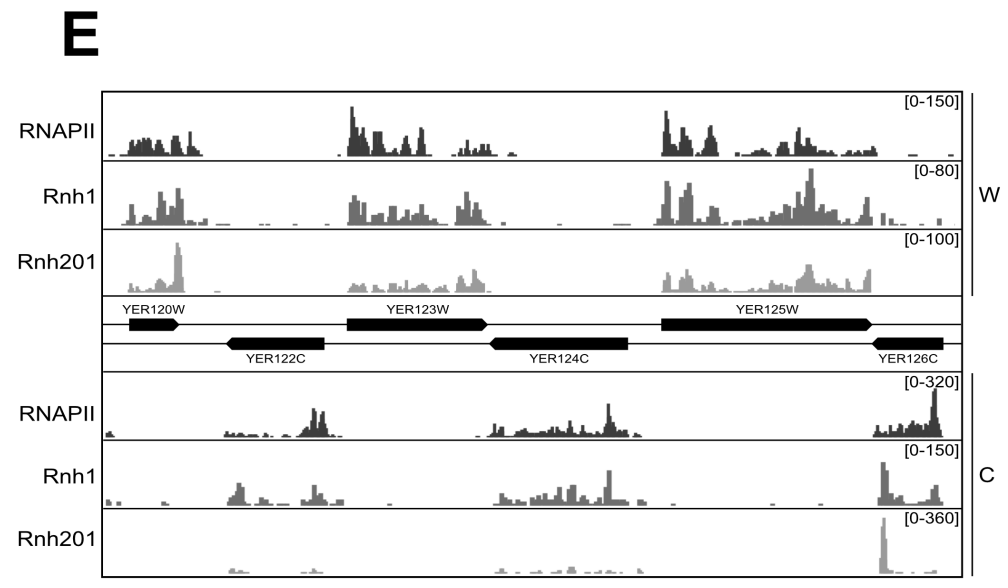

G
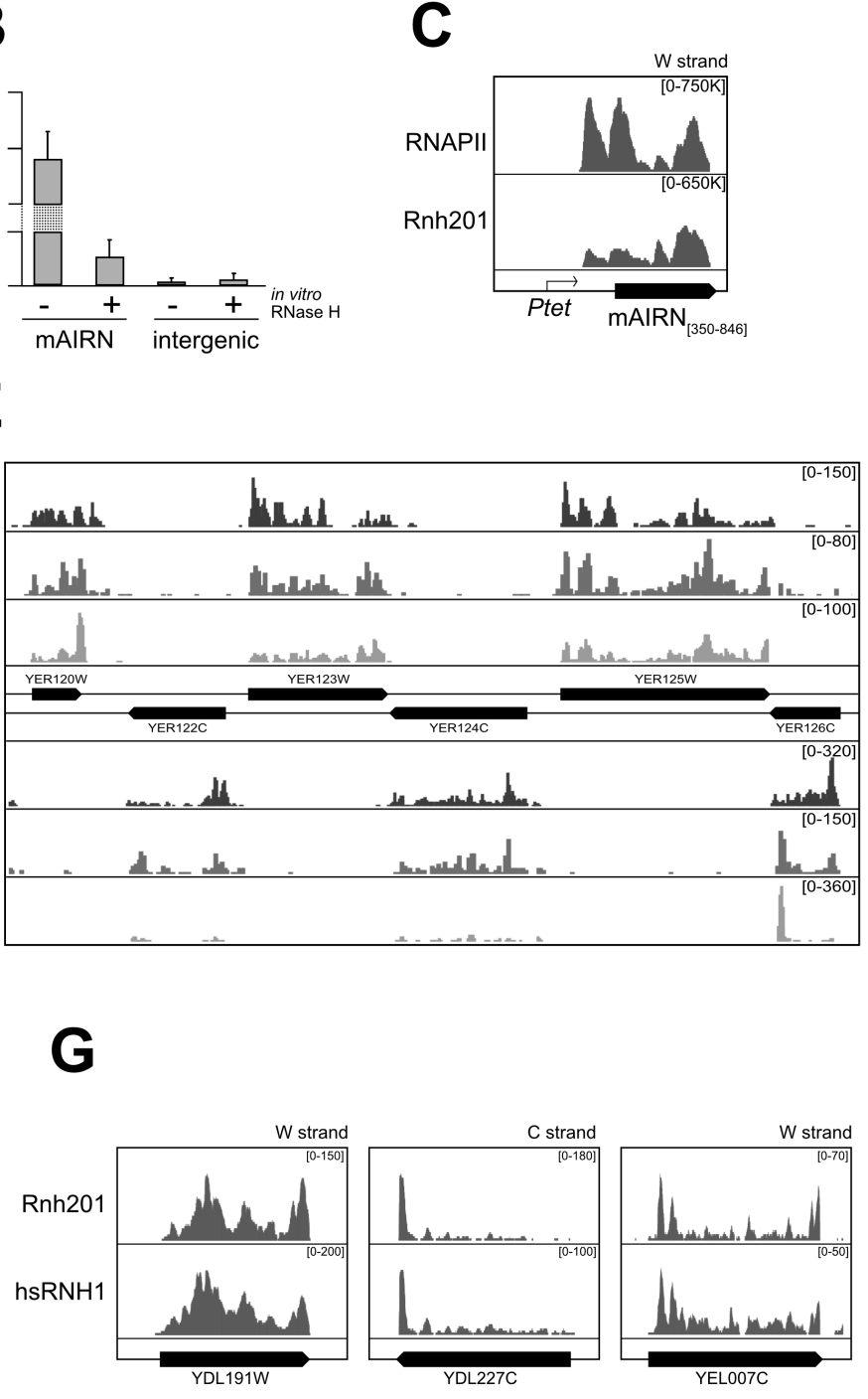


\section{Supplementary Figure 3 related to Figure 4:}

A) Dispersion plot of the $\log _{2}$ values obtained from Rnh1 H-CRAC and Rnh201 H-CRAC for the 3000 most transcribed genes in asynchronous cells. The coefficient of determination $\left(r^{2}\right)$ is shown. B) DNA:RNA Immunoprecipitation followed by quantitative PCR (DRIP-qPCR) from cells transformed with a plasmid carrying the mAIRN sequence expressed under control of the pTet promoter as indicated on the scheme in panel C (only the R-loop-forming region corresponding to the $350-848 \mathrm{nt}$ interval of the mouse gene was cloned). The percentage of immunoprecipitated material is plotted on y-axis. The DRIP signal from an intergenic region located nearby to the $\mathrm{HO}$ locus was used as a negative control. Samples were treated or not with $\mathrm{RNase} \mathrm{H}$ in vitro prior to immunoprecipitation as indicated. C) Read coverage for RNAPII (CRAC) and Rnh201 (H-CRAC) on the plasmid-borne mAIRN sequence. D) Dispersion plots illustrating the correlation between transcription in genes (RNAPII CRAC) and R-loop levels, as determined by H-CRAC (top: Rnh1; bottom: Rnh201). E) Integrative Genomics Viewer (IGV) representative screenshot of a chromosomic region illustrating the marked directionality of $\mathrm{H}-\mathrm{CRAC}$ signals for both Rnh1 and Rnh201 as indicated. F) Metagene analyses of RNAPII CRAC signal at coding genes aligned on their TSS and on their pA site in wild-type (WT) cells transformed with a plasmid overexpressing hsRNH1 (+) or an empty plasmid (-). Genes are only scaled in between the red lines. G) Representative snapshots illustrating the similarities between the H-CRAC signal obtained with yeast Rnh201 or human RNH1 (hsRNH1). H) Dispersion plot as in Figure S3A but comparing Rnh201 to hsRNH1. 


\section{Figure S4 related to Figure 4}

\section{A}
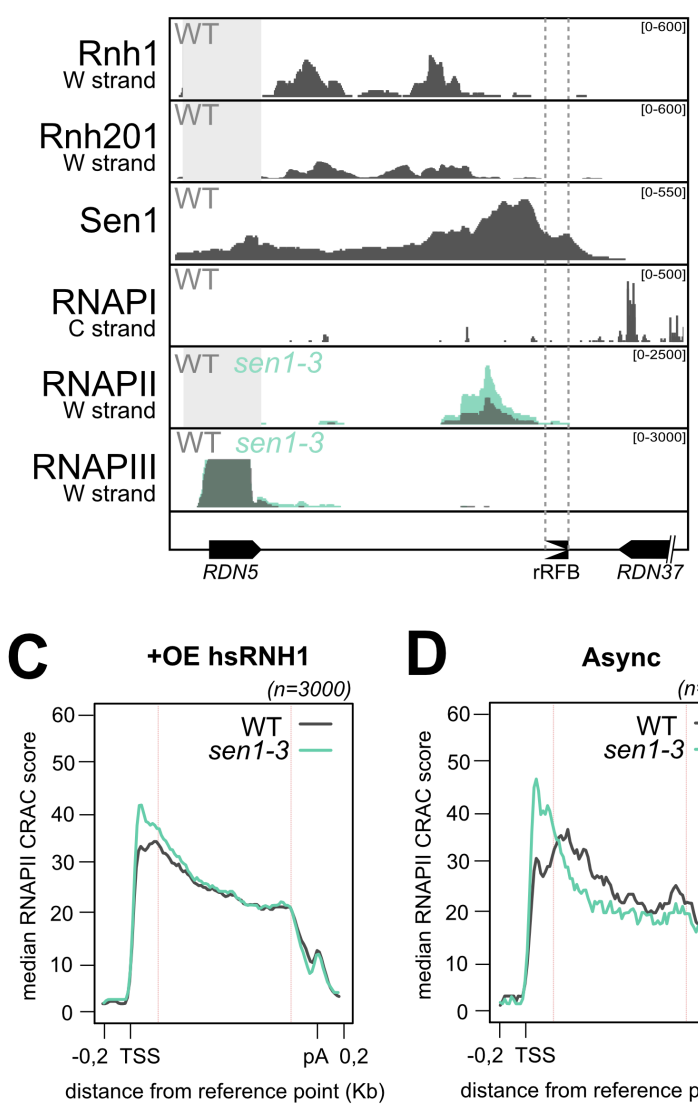

G

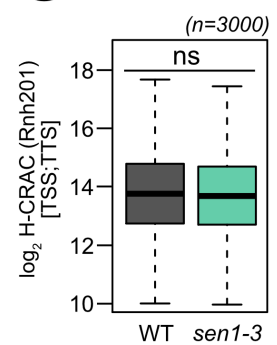

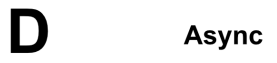

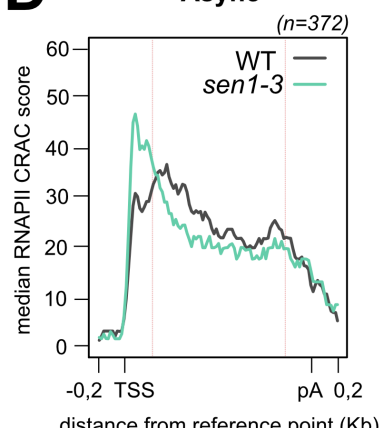

$\mathbf{E}$

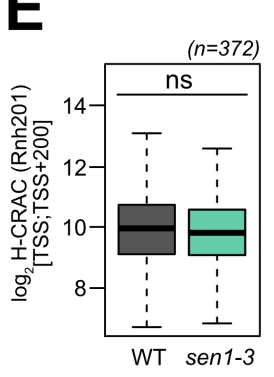

$\mathbf{F}$

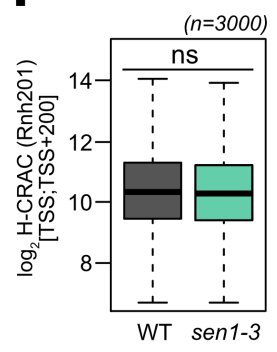

\section{Supplementary Figure 4 related to Figure 4:}

A) Distribution of CRAC signals with the indicated proteins at the rRFB. Data for RNAPI (Turowski et al., 2020) and RNAPIII (Xie et al., 2021) indicate that accumulation of RNAPII is unlikely due to conflicts with RNAPI or RNAPIII. Sen1 ChIP-exo signals (Rossi et al., 2021) demonstrate the presence of Sen1 at the site of TRC. The strand shown is indicated for each protein, with the exception of Sen1 because ChIP data are not directional. B) Metagene analyses of RNAPII CRAC signal at coding genes aligned on their TSS and PA site in rnh14 


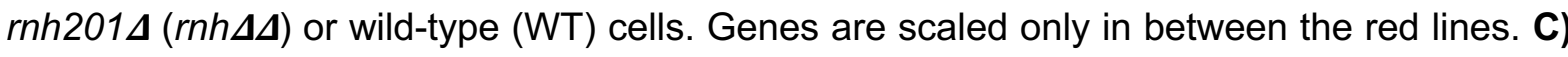
As in Figure S4B but for WT and sen1-3 cells transformed with a plasmid over-expressing human RNH1. Note that the 5'-end RNAPII accumulation observed in sen1-3 cells is not lost in these conditions. D) As in Figure $2 \mathrm{C}$ but on asynchronously dividing cells and on the subset of genes affected in this condition. E) and F) Boxplot comparisons of the H-CRAC signal on the interval [TSS; TSS+200] for the same group of genes shown in Figure S4D or for the most transcribed 3000 genes, respectively. G) As in Figure S4F, but for the signal along the full gene [TSS; TTS]. H) Growth assay of sen1-3 rat1-1 cells compared to single mutants. Serial dilutions of the indicated strains were incubated for 3 days at the indicated temperature. Growth was performed on the same plates. 


\section{Figure S5 related to Figure 5}
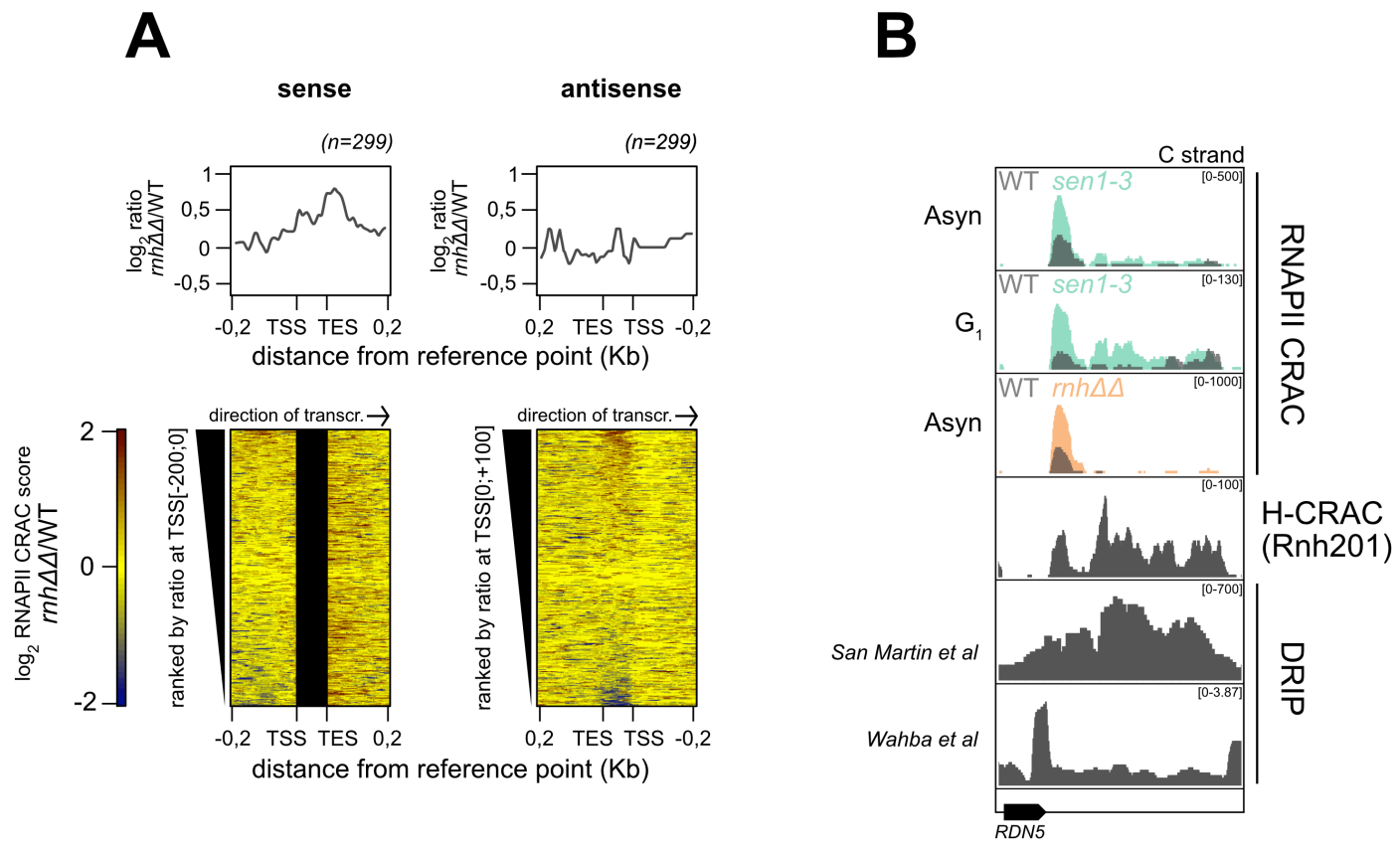

\section{Supplementary Figure 5 related to Figure 5:}

A) Heatmap analyses representing the $\log _{2}$ ratio ( $r n h 1 \Delta$ rnh2014 WT) of the RNAPII CRAC signal at tRNA genes aligned at their Transcription Start Site (TSS) both for the sense and the antisense transcription as indicated. Genes were ranked as in Figure 5B. The summary plot on the top was calculated using the median values foreach position. B) Accumulation of RNAPII antisense to the RDN5 gene in WT, sen1-3 and rnh14 rnh2014 cells as in Figure 5A for tRNAs. R-loops levels from H-CRAC and DRIP-seq (San Martin-Alonso et al., 2021; Wahba et al., 2016) are also shown. 


\section{Figure S6 related to Figure 6}

A

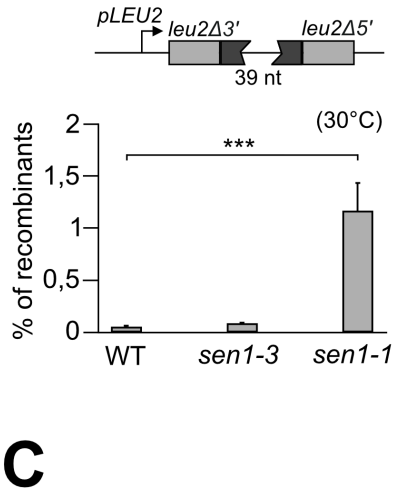

$\left(30^{\circ} \mathrm{C}\right)$
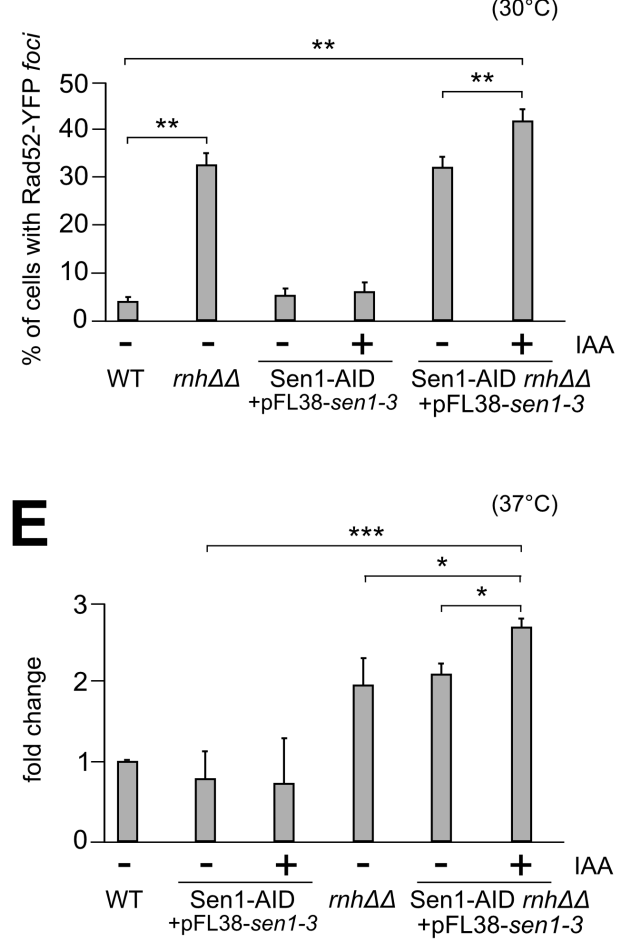

B

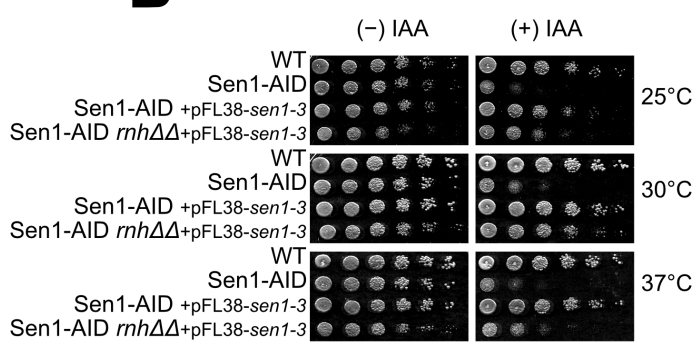

D

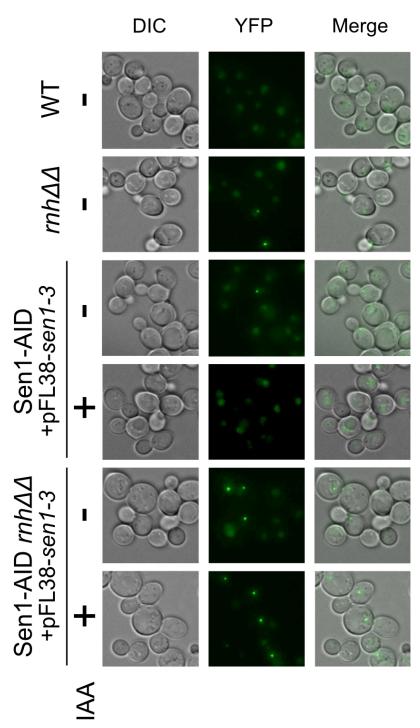

\section{Supplementary Figure 6 related to Figure 6:}

A) Frequency of transcription-associated recombination (TAR) events assessed using the $\mathrm{pL}$ (Prado and Aguilera, 2005) reporter in wild-type (WT), sen1-3 and sen1-1 cells. The reporter plasmid (schematised above the graph) contains the LEU2 gene interrupted by a $39 \mathrm{nt}$ insertion flanked by homology repeats. Transcription activation in the absence of leucine induces damage and recombination between the two repeats, which reconstitutes a functional 
LEU2 gene. ${ }^{* * *} p<0.001$ B) Growth assay of inducible triple mutant used for the analyses shown in Figures 6 and S6. Note that the establishment of the phenotype is partial, possibly due to the partial depletion of Sen1 or a suppression effect of slight Sen1-3 overexpression. C) As in Figure 6B, but growth was performed at $30^{\circ} \mathrm{C}$. ${ }^{* *} \mathrm{p}<0.01$. D) Representative examples of the microscopy data shown in Figure S6C. E) Quantification of H2A Ser129 phosphorylation detected by western blot in Figure 6C. Cells were grown in logarithmic phase at $30^{\circ} \mathrm{C}$, and then shifted at $37^{\circ} \mathrm{C}$ for 1 hour. The fold change relative to the WT levels is shown for the indicated conditions. Pab1, Nrd1 and Nab3 were used as loading controls. Error bars represent standard deviations. ${ }^{*} p<0.05 ;{ }^{* *} p<0.001$. 


\section{Figure S7}
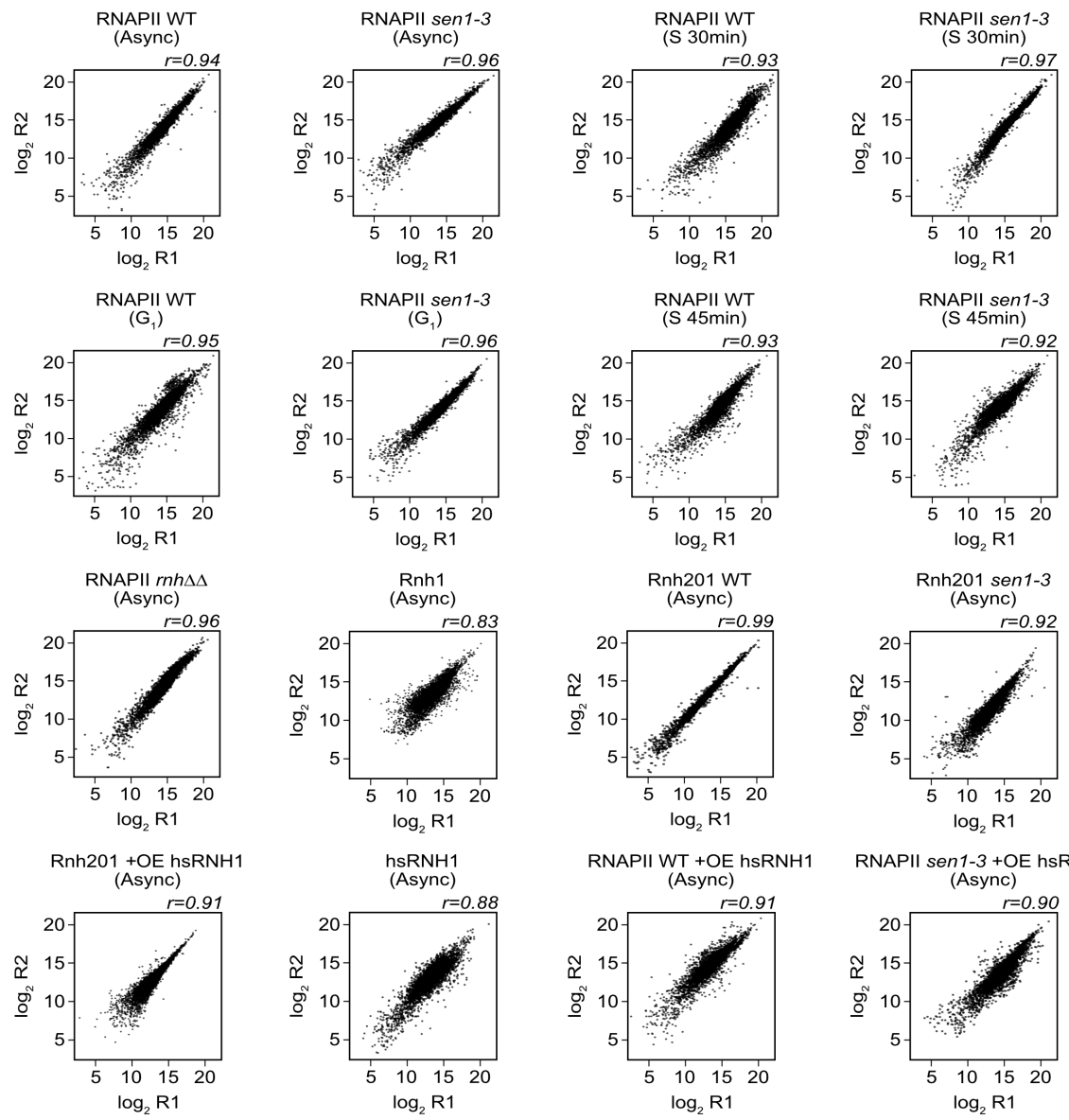

\section{Supplementary Figure 7:}

Correlation plots between replicates of the CRAC experiments shown in this study. The Pearson correlation score $(r)$ is shown at the top right of each plot. 